\title{
On the Perturbation of the Three-Dimensional Stokes Flow of Micropolar Fluids by a Constant Uniform Magnetic Field in a Circular Cylinder
}

\author{
Panayiotis Vafeas, Polycarpos K. Papadopoulos, \\ and Pavlos M. Hatzikonstantinou \\ Department of Engineering Sciences, University of Patras, 26504 Patras, Greece \\ Correspondence should be addressed to Panayiotis Vafeas, vafeas@des.upatras.gr
}

Received 3 June 2010; Accepted 25 January 2011

Academic Editor: Alexander P. Seyranian

Copyright (C) 2011 Panayiotis Vafeas et al. This is an open access article distributed under the Creative Commons Attribution License, which permits unrestricted use, distribution, and reproduction in any medium, provided the original work is properly cited.

\begin{abstract}
Modern engineering technology involves the micropolar magnetohydrodynamic flow of magnetic fluids. Here, we consider a colloidal suspension of non-conductive ferromagnetic material, which consists of small spherical particles that behave as rigid magnetic dipoles, in a carrier liquid of approximately zero conductivity and low-Reynolds number properties. The interaction of a 3D constant uniform magnetic field with the three-dimensional steady creeping motion (Stokes flow) of a viscous incompressible micropolar fluid in a circular cylinder is investigated, where the magnetization of the ferrofluid has been taken into account and the magnetic Stokes partial differential equations have been presented. Our goal is to apply the proper boundary conditions, so as to obtain the flow fields in a closed analytical form via the potential representation theory, and to study several characteristics of the flow. In view of this aim, we make use of an improved new complete and unique differential representation of magnetic Stokes flow, valid for nonaxisymmetric geometries, which provides the velocity and total pressure fields in terms of easyto-find potentials. We use these results to simulate the creeping flow of a magnetic fluid inside a circular duct and to obtain the flow fields associated with this kind of flow.
\end{abstract}

\section{Introduction}

Many chemical, biochemical, and other industrial or biological processes employ solid or soft matter in the form of small ferromagnetic particles, which are embedded in a Newtonian fluid and react in the presence of a magnetic field. Several engineering applications with high technical complexity that cover the large specific area that is offered by such systems have an inherent interest of physical and mathematical nature [1]. Those systems involve the pure hydrodynamic motion of the aforementioned aggregates of particles, where the application of a magnetic field perturbs the flow and the coexistence of liquid and magnetic properties provides us with useful information on the physical problem that has to be solved in each 
case. It is to this end that magnetic fluids [1], such as water, hydrocarbon, ester, fluorocarbon, and others, comprise a novel class of engineering materials, where their specific magnetic and hydrodynamic feature promotes heat and mass transfer, reaction rates, blood or other biological flows, and so forth, under the action of magnetic fields. More specifically, magnetic fluids can be considered as colloidal suspensions of spherical particles in a conductive liquid, where the assumption of complete isotropic shape of the magnetic particles is valid due to their small size. In this case, the so-called ferromagnetic particles follow the Brownian motion and behave as rigid magnetic dipoles. Thus, the application of an external magnetic field, apart from the creation of an induced magnetic field of minor significance, will prevent the rotation of each particle, increasing the effective viscosity of the fluid and will cause the appearance of an additional magnetic pressure. The combination of the hydrodynamic flow [2] with the application of an arbitrarily orientated magnetic field allows us to consider the fluid as micropolar $[1,3,4]$ and provides us with the appropriate tools for developing and solving such boundary value problems of high mathematical and technical complexity. The most general consideration secures the consistency of many boundary value problems with the principles of both ferrohydrodynamics and magnetohydrodynamics, by including, respectively, both magnetization and electrical conductivity of the fluid within the partial differential equations, which are under analytical or numerical investigation [14]. Specifically, ferrohydrodynamics is concerned with the mechanics of the motion of the so-called micropolar fluids that is influenced by strong forces of magnetic polarization, introducing a magnetic stress tensor [5] into the momentum equation. On the other hand, magnetohydrodynamics deals with the current distribution of the electrically conductive carrier liquids. It is worth mentioning that nowadays a very big part of the scientific research is dedicated to the biomagnetic fluid dynamics, which combines the existence of the magnetization of the biofluid with its conductivity; see, for instance, blood flow problems. The system of hydrodynamic equations that governs the magnetic fluids flow [1$4]$ is constituted by the equations of continuity, of momentum, of Maxwell, of energy (when we deal with the thermomechanics of a magnetic fluid [3]), of angular momentum, and of magnetization, which are all coupled with each other. Those partial differential equations are expressed in terms of the velocity field, the total pressure field, the variation of the temperature, the magnetic field, the conduction current density, and the several specific hydrodynamic or magnetic parameters, which characterize the ferrofluid and the motion itself. The appropriate boundary conditions, depending on the particular physical problem, are adequate for the completeness of a well-posed boundary value problem.

Recently, a general three-dimensional theoretical model that conforms to physical reality and at the same time permits the analytical investigation of the aforementioned partial differential equations has been developed by Hatzikonstantinou and Vafeas [6]. In this novel model the authors focused both on ferrohydrodynamic and on magnetohydrodynamic problems of technological or biomechanical interest, which can be applied to various micropolar magnetic fluids, by constructing purely analytical solutions and using the minimum of the necessary assumptions for their model. This work [6] involves the study of the incompressible flow of a Newtonian carrier magnetic liquid, which contains a small concentration of magnetic particles under the effect of an arbitrarily orientated applied magnetic field. There, it has been presented the development of a new dyadic, as well as vector, expression for the momentum equation, which incorporates an explicit theoretical expression for the extra viscosity. The additional magnetic pressure generated by the effect of the magnetic field and a general three-dimensional analytical expression for the magnetization and the Lorentz forces are also included. However, many of the industrial 
or biological systems that we have already described consider pipe flow problems, which can be modeled by the Stokes flow [7] approximation in the presence of a magnetic field, which involve the creeping motion of magnetic fluids in low Reynolds number [7]. Therefore, the aforementioned partial differential equations reduce to a simpler shape that is similar to the well-known Stokes equations [7], the so-called magnetic Stokes equations, which are presented in every detail in [6]. Within the frame of the already known potential representation theory developed in early years [8-10], a new complete and unique differential representation of magnetic Stokes flow has been constructed [6], valid for nonaxisymmetric geometries, which provides the velocity and total pressure fields in terms of easy-to-find potentials, via an analytical fashion. This representation considers steady state, neglecting the current distribution in the fluid and approximating the magnetization by its equilibrium expression (more specifically by the constant saturation expression for extremely strong magnetic fields at very low temperatures). These two last assumptions arise from the small size or the low concentration of the ferromagnetic particles neglecting magnetorestrictive effects and from the fact that, for creeping flow, very low velocities are considered. Such an analytical approach of creeping flow (Stokes flow) under the action of a magnetic field, arbitrarily orientated, in three-dimensional interior and exterior flow fields, appears for the first time, and, as it will be shown later, it is the key to our work.

A very large part of the applications in the technological field and in general biomagnetic processes involves the micropolar flow of magnetic fluids inside pipes or ducts and their reaction to the presence of magnetic fields. An approach of one-dimensional case appears in [1] by Berkovski and Bashtovoy, where it is examined under various strict conditions the theoretical analysis of a steady laminar flow in a cylinder under the action of an axial or transverse magnetic field, depending only on the axial direction of the flow. Similar simplified solutions like the problem of a plane Couette flow have been also developed by Rosensweig in [4], while an interesting analytical work by Verma [11] on ferrohydrodynamics deals with a two-dimensional flow in Cartesian coordinates, introducing the well-known Stokes stream function [2]. Although the study of the reaction of micropolar fluids when they are disturbed by several types of magnetic fields is of great interest, it still remains useful to report papers concerning the flow of fluids with micropolar properties inside pipes or around bodies, where some works retain and others omit (considering creeping flow) the convection terms in the Navier-Stokes equations. Recently, a pure analytical solution was obtained by Sastry and Mohan Rao [12], who discussed the micropolar fluid flow arising due to oscillations of a plane, when the system is subject to uniform rotation, whilst a hybrid paper of Calmelet-Eluhu and Majumdar [13] investigates the internal flow of a micropolar fluid inside a circular cylinder, which is subject to longitudinal and torsional oscillations, where the obtained analytical results are followed by numerical analysis. Under this aspect, Weng et al. [14] employed the theory of micropolar fluids in order to study the stability problem of flow between two concentric rotating cylinders, providing analytical and numerical results as well. On the other hand, Stokes flow in micropolar fluids appears frequently in the recent years, providing interesting information about the creeping flow of magnetic fluids. Indeed, Faltas and Saad [15] examined the axisymmetric Stokes flow of a sphere bisected by a free surface bounding a semi-infinite micropolar fluid, and three years later Sherif et al. [16] presented the Stokes axisymmetrical flow caused by a sphere translating in a micropolar fluid perpendicular to a plane wall at an arbitrary position from the wall. In addition, the same year, Moosaie and Atefi [17] provided an analytical solution for the creeping flow of a micropolar fluid past a rotating circular cylinder of infinite length in spanwise direction. Nevertheless, those 
analytical or semianalytical works introduce several physical or mathematical assumptions and conditions in order to overcome certain difficulties arisen by the particular analytical investigation. Those constraints multiply rapidly when someone has to face the problem of the interaction of micropolar fluids with magnetic fields and then the difficulties increase exponentially. Hence, the use of purely numerical methods was obligatory. Within this frame, two important works by Papadopoulos and Tzirtzilakis [18] as well as Khashan and Haik [19] are involved with the biomagnetic fluid dynamics under the action of a magnetic field. Moreover, in the aim of investigating the reaction of liquid metals to magnetic fields, several authors have taken into account the electric conductivity of such liquids. Under this aspect, two works by Sellers and Walker [20] and Witkowski and Walker [21] have used the principles of magnetohydrodynamics in order to construct numerical solutions for the flow of liquid metals, taking into account the electric current density. Recently, Papadopoulos et al. [22] studied numerically a first approach of the new theoretical model presented in [6] by examining the magnetohydrodynamic flow of a micropolar magnetic fluid in a straight square duct. Under this point of view, Vafeas et al. [23] provided analytical and numerical results about the flow of a micropolar fluid under the effect of a line dipole. However, all of these methods involve the use of elaborate computer codes for each case under consideration. But there is always room and need for strong analytical methods, which incorporate the appropriate geometrical and physical characteristics with the minimum of the simplifications. It is to this end that Stokes flow serves as the platform of the necessary assumptions that have to be made in order to keep consistency between the mathematics and the physics in real flow situations. During the last century, several authors have employed the creeping flow [7] in order to solve the corresponding physical boundary value problems. For example, such a large area of applications concerns the well-known particle-in-cell models for the Stokes flow through relatively homogeneous swarm of particles (inorganic, organic, biological). Those systems provide a relatively simple platform for the analytical or semianalytical solution of heat or mass transport problems. Two reference papers by Dassios et al. [24] and Vafeas and Dassios [25] on this matter involve the solution of such problems in axisymmetric spheroidal and in 3D ellipsoidal coordinates, respectively. More specific, in [25] the analytical solution for the flow fields has been obtained with the aim of the PapkovichNeuber differential representation $[8,10]$, which was the motivation for the construction of a new differential representation for magnetic Stokes flow [6].

Until nowadays, one can rarely retrieve reports of works in the literature that capture in an analytical fashion the influence of magnetic fields to flows of micropolar or electrically conductive fluids, where the complete anisotropy of the three-dimensional space must be seriously taken into account. For example, Martin Witkowski et al. [26] treated the nonaxisymmetric flow of an electrically conducting liquid in an insulating cylinder with a spatially uniform, transverse, rotating magnetic field. Here, in this paper, we obtain the analytical solution to the fully three-dimensional Stokes flow problem of a micropolar fluid with low Reynolds number properties (neglect of the convection terms in the Navier-Stokes equations) inside a circular cylinder, where the flow is perturbed by a constant vector uniform magnetic field that is arbitrarily orientated. In order to obtain the flow fields, we use a differential representation, which is based on the one developed in [6], and it provides the velocity and the total pressure of the fluid in terms of easy-to-find potentials that contain the magnetic field. The representation used here considers the same assumptions made in [6], namely, steady state flow and no current distribution in the fluid. The main improvement of the present representation compared to the one in [6] is that it approximates the magnetization by the more general equilibrium expression which contains the measure 
of the general applied magnetic field and not by the constant saturation expression. Thus, it has increased accuracy and more general applicability without being more complicated than the one in [6]. An outline of the differential representation that we employ as well as the additional steps needed for the present improved representation of the flow fields is presented in a later section.

Hence, under the assumption of low Reynolds number and small size or low concentration of ferromagnetic particles in the micropolar fluid, the general differential solution provides the velocity and the total pressure fields via potentials that satisfy easy-handled equations, which contain the applied magnetic field. For a constant magnetic field in the three dimensions as in our case, those equations reduce to Laplace equations for each potential function. Consequently, the unknown potentials are given via infinite series expansions of internal harmonic eigenfunctions in circular cylindrical coordinates [27-29], since we face an interior flow problem inside a circular cylinder, and, then, the 3D flow fields are provided via closed forms of full series expansions through the differential representation. That way, the generality of the potentials is inherited to the magnetic flow fields, via a complete set of unknown constant coefficients that have to be calculated explicitly. In order to achieve that, we consider a circular cylinder of a finite length for our problem, and we supplement the general solution with the appropriate boundary conditions as a fair approximation for the creeping flow of a micropolar fluid inside a finite circular cylinder in the presence of a constant magnetic field. These conditions are nonslip condition on the wall of the cylinder, an imposed axial velocity of a known general form with no transversal components at the entrance of the cylinder, and cancellation of the axial derivatives of the transversal components of the velocity as well as zero axial stresses at the exit of the cylinder. We point out that the last condition has been introduced by Gresho [30] and Ganesh [31]. In order to secure consistency with the physical requirements, those conditions are supplemented with the demand of conservation of mass at the edge of the finite cylinder (Bruneau and Fabrie [32]). All the boundary conditions reported here are extensively used in many papers, where their basic characteristics are concentrated within the references [30-32]. Applying the conditions to the general differential solutions for the flow fields, we perform many tedious and long calculations in order to evaluate the unknown constant coefficients and obtain the velocity and the total pressure in a closed analytical form of expansions of infinite series, in terms of the applied 3D constant magnetic field, of the interior circular cylindrical eigensolutions, and of the certain hydrodynamic or magnetic parameters. In addition, we accomplish to reveal the effect of the applied magnetic field on the magnetic Stokes flow fields and on the viscosity of the fluid, where its increase is clearly shown by a factor, which is the ratio of the apparent viscosity of the flow over the hydrodynamic viscosity of the fluid. The aforementioned closedtype solution is general and valid for the nonaxisymmetric circular cylindrical geometry, and all the details as well as the difficulties arisen during the calculation process are discussed extensively in the corresponding section of the paper. Here, we add that our calculations were made within the classical mathematical analysis framework using reliable bibliography $[27-29,33]$, while a mathematical technique based on a theory for the completeness of the differential representations by Eubanks and Sternberg [34] (also used in [25]) was imposed.

The analytical section of the present paper is followed by the application of the obtained solution to the computation of the velocity and total pressure fields of creeping flow in a circular duct. The results include plots that depict the development of the flow as the magnetic fluid moves downstream of the duct, under the effect of the imposed magnetic field. We also prove that the additional friction losses depend upon the effective viscosity of the flow [35]; hence, we present plots that depict the variation of the additional viscosity due 
to the magnetic field versus several characteristic parameters of the flow, such as the magnetic field magnitude and the concentration of particles inside the ferrofluid.

Finally, the necessary mathematical material from the theory of harmonic functions in circular cylindrical coordinates and the Bessel functions as well as some useful formulae associated with trigonometric and hyperbolic functions is collected in the appendix, along with the presentation of various vector identities.

\section{Mathematical Formulation and the New Differential Representation for Magnetic Stokes Flow}

In order to formulate our boundary value problem, we consider a finite circular cylinder of radius $\alpha$ and of length $L$, and, under steady state conditions with no time dependence, we examine the interior creeping flow (very small Reynolds number [7]) of an incompressible (constant mass density $\rho$ ) and viscous (constant dynamic viscosity $\eta$ ) micropolar fluid inside the smooth bounded three-dimensional cylindrical domain $V\left(\mathbb{R}^{3}\right)$ that is confined by the cylinder's prescribed dimensions. In what follows, every field will be written in terms of the position vector $\mathbf{r}=x_{1} \widehat{\mathbf{x}}_{1}+x_{2} \widehat{\mathbf{x}}_{2}+x_{3} \widehat{\mathbf{x}}_{3}$ expressed via the Cartesian basis $\widehat{\mathbf{x}}_{i}, i=1,2,3$, in Cartesian coordinates $\left(x_{1}, x_{2}, x_{3}\right)$, where this dependence will be omitted for convenience in writing. This coordinate system is placed in such a way so as the $x_{1}$-axis follows the direction of the axis of symmetry of the finite circular cylinder, while the other two axes lie on the perpendicular plane. On the other hand, its connection with the appropriate $(r, \varphi, z)$ circular cylinder coordinate system is provided in the appendix via relation (A.12). Micropolar fluids

[1] are characterized by colloidal suspension of very small solid ferromagnetic particles, which are considered as spherical of radius $r_{p}$ and of density $\rho_{p}$ due to their minor size and are finely divided in a continuous liquid medium that is considered nonconductive (without any Lorentz force density $[1,6]$ ) in our case. This last hypothesis conforms to physical reality, since the volumetric concentration of the ferromagnetic particles remains in low levels, and, then, the current distribution is negligible. The ferrofluid consists of the carrier fluid and the suspension of magnetic particles, which behave as rigid magnetic dipoles. The particles do not interact to form agglomerations due to the Brownian motion, which is responsible for the stability of the ferrofluid. Hence, the application of an external magnetic field will prevent the rotation of each particle, increasing the effective viscosity of the fluid, and will change the total pressure.

Under the aforementioned assumptions, the governing micropolar hydrodynamic equations of our physical problem relate the velocity field $\mathbf{v}$ and the total pressure field $P=p+\rho g x_{3}$ with the applied magnetic field $\mathbf{H}$ of measure $H=|\mathbf{H}|$. It is noted that $p$ is the thermodynamic pressure, $\mathbf{g}=-g \widehat{\mathbf{x}}_{3}$ defines the acceleration of the gravity of measure $g$, and $\rho g x_{3}$ refers to a hydrostatic pressure force, which corresponds to a height of reference in the $x_{3}$-direction. Moreover, the induced magnetic field is taken approximately equal to zero, which is true in many applications. The governing equations are the Stokes magnetohydrodynamic equation for the creeping motion of magnetic fluids $[1,4,6]$

$$
\left[\eta+\frac{\tau_{B} \mu_{0} M_{0} H}{4\left(1+\left(\tau_{S} / I\right) \tau_{B} \mu_{0} M_{0} H\right)}\right] \Delta \mathbf{v}=\nabla P-\mu_{0} M_{0} \nabla H
$$

and the continuity equation

$$
\nabla \cdot \mathbf{v}=0
$$


which secures the incompressibility of the ferrofluid. They are both expressed in terms of the well-known differential operators $\nabla$ and $\Delta$ (see also (A.15)). Here, $\tau_{S}=r_{p}^{2} \rho_{p} / 15 \eta_{0}$ is the relaxation time of particle rotation ( $\eta_{0}$ corresponding to the rotational viscosity), $I=8 \pi r_{p}^{5} \rho_{p} n / 15$ is the sum of moments of inertia of the spherical particles per unit volume ( $n$ being the number of particles per unit volume), $\tau_{B}=4 \pi \eta r_{p}^{3} / K T$ is the relaxation time of Brownian rotation ( $K$ being the Boltzmann's constant and $T$ denoting the temperature, which we consider as constant for the present isothermal problem), and $\mu_{0}$ is the magnetic permeability of the free space. Furthermore, as it is clearly explained in [6], for creeping flow, as in our case, small velocities are obtained, and then the magnetization $\mathbf{M}$ (of measure $M=|\mathbf{M}|$ ) becomes approximately colinear with $\mathbf{H}$. Their connection is provided via the equilibrium magnetization $M_{0}$ and in terms of the Langevin function $L(\xi)$ by the equation

$$
\mathbf{M} \cong \frac{M_{0}}{H} \mathbf{H} \Longrightarrow M \cong M_{0}=n m L(\xi) \quad \text { for } L(\xi)=\operatorname{coth} \xi-\frac{1}{\xi} \text { with } \xi=\frac{\mu_{0} m H}{K T}
$$

where $m$ specifies the magnetic moment of a particle, while $M_{0}=M_{0}(H)$. It is obvious that, in some cases, we have to take into consideration the magnetization of the particle itself $M_{p}$, which is connected with the saturation magnetization $M_{s}$ by the relation

$$
M_{s}=\phi M_{p}=n m \quad \text { with } \phi=\frac{4}{3} \pi r_{p}^{3} n
$$

where $\phi$ is the volumetric concentration of particles. On the other hand, as $\xi \rightarrow 0$, then $L(\xi) \rightarrow 0$, whilst as $\xi \rightarrow+\infty$, it is easily verified that $L(\xi) \rightarrow 1$. Hence, $M_{0}$ can sometimes be approached by the constant saturation expression for extremely strong magnetic fields at very low temperatures. The vorticity of the fluid $\Omega$ (of measure $\Omega=|\Omega|$ ) is expressed as

$$
\boldsymbol{\Omega}=\nabla \times \mathbf{v}
$$

The quantity $\Omega \tau_{B}$ stands for the crucial characteristic that controls the nature of the micropolar flow. For instance, Stokes micropolar flow is confirmed by the condition $\Omega \tau_{B} \ll 1$ that reflects small velocities. For convenience to our calculations, we define the dimensionless parameter $\chi(H)$ as

$$
\begin{aligned}
x(H) & \equiv \frac{1}{\eta}\left[\eta+\frac{\tau_{B} \mu_{0} M_{0} H}{4\left(1+\left(\tau_{S} / I\right) \tau_{B} \mu_{0} M_{0} H\right)}\right] \\
& =\left[1+\frac{\tau_{B} h(H)}{4 \eta\left(1+\left(\tau_{S} / I\right) \tau_{B} h(H)\right)}\right] \text { for } h(H)=\mu_{0} M_{0} H,
\end{aligned}
$$

which is the ratio of the total viscosity of the micropolar fluid in the presence of magnetic particles that can respond to a magnetic field over the viscosity of the fluid in the absence of magnetic particles. The term

$$
\frac{\tau_{B} \mu_{0} M_{0} H}{4\left(1+\left(\tau_{S} / I\right) \tau_{B} \mu_{0} M_{0} H\right)}=\eta(x(H)-1)
$$


appearing in (2.1) is the additional effective viscosity caused by the interaction between the magnetic particles and the applied magnetic field. Inserting definitions (2.6) into the momentum equation for creeping magnetic flow (2.1), we obtain the equivalent form

$$
\eta \chi(H) \Delta \mathbf{v}=\nabla P-h(H) \nabla(\ln H)
$$

The function $h(H)$, which is defined in (2.6), stands for the effect of the Brownian motion of the particles on the total pressure. If there are no magnetic particles $(n=\phi=0)$ or if there is no magnetic field $(H=0)$, then from (2.3) we have $M_{0}=0$, which means that $h(H)=0$ and, consequently, $\chi(H)=1$ as it is revealed from relation (2.6). Then, the pair of relationships (2.8) and (2.2) reduces to the already known Stokes equations for the hydrodynamic creeping flow [7].

Our first purpose is focused on the analytical treatment of the coupled equations of momentum (2.8) and continuity (2.2). Under this aspect we search for analytical solutions of these equations in the forms of differential representations [8-10], which provide us with the velocity field $\mathbf{v}$ and the total pressure field $P$ in terms of differential operators that act on particular potentials and in terms of the applied magnetic field $H$. Within this frame, in publication [6], an extended paragraph with a proved theorem was devoted to that matter in the case where the equilibrium magnetization is taken nearly equal to the constant saturation magnetization $\left(M_{0} \cong M_{s}=n m\right)$. Nevertheless, in this paper it is our goal to extend this theorem in the case where the equilibrium magnetization is provided by its most general expression (2.3), which contains the measure of the general applied magnetic field. In view of that, we present the following improved differential representation for magnetic Stokes flow, which provides the velocity and the total pressure of the fluid in terms of easy-to-find potentials that contain the magnetic field, in the more general case, where $M_{0}$ is given by (2.3); that is,

$$
\begin{gathered}
\mathbf{v}=\mathbf{\Phi}-\frac{1}{2} \nabla(\mathbf{r} \cdot \mathbf{\Phi}+\Psi), \\
P=[h(H)-\bar{h}(H)]-\eta \chi(H) \nabla \cdot \boldsymbol{\Phi}+P_{0} \quad \text { with } \nabla \bar{h}(H)=h(H) \nabla\left(\ln M_{0}\right),
\end{gathered}
$$

where $P_{0}$ is a constant reference pressure and $\Phi, \Psi$ are potentials that satisfy

$$
\Delta \mathbf{\Phi}+\nabla[\ln \chi(H)] \nabla \cdot \boldsymbol{\Phi}=\mathbf{0}, \quad \Delta \Psi=-\mathbf{r} \cdot \Delta \mathbf{\Phi},
$$

respectively. The representation (2.9)-(2.11) is the new, more complete, general solution that we present for the first time in this paper, which provides us with the magnetic flow fields of (2.8) and (2.2) in an analytical fashion. If there are no magnetic particles $(n=\phi=0)$ or if there is no magnetic field $(H=0)$, we obtain $M_{0}=0, h(H)=\bar{h}(H)=0, x(H)=1$, and our differential representation (2.9)-(2.11) reduces to the already known general solution for Stokes flow [10]. The proof of completeness and of uniqueness of the differential solution (2.9)-(2.11) follows the same steps provided in [6] by making the change $h_{[6]}(H) \rightarrow[h(H)-\bar{h}(H)]$. Thus, in order to avoid repetition we choose to omit the proof and refer to [6] for further analysis and elaboration. However, it is not difficult to show that solution (2.9) and (2.10) 
with (2.11) satisfy (2.8) and (2.2). Indeed, with the aid of identity (A.1) we transform the momentum equation (2.8) to

$$
\eta \chi(H) \Delta \mathbf{v}=\nabla(P-h(H))+h(H) \nabla\left(\ln M_{0}\right)
$$

Utilizing identity (A.11) and substituting the velocity (2.9) and the total pressure (2.10) into (2.2) and (2.12), then the last two are immediately satisfied, taking into account the relationships for the potentials (2.11).

Our final task is to find an analytical form for $\bar{h}(H)$, whose gradient appears in (2.10). In order to achieve this, we proceed as follows. We make use of (2.3), (2.6), and (2.10) to calculate

$$
\nabla \bar{h}(H)=h(H) \nabla\left(\ln M_{0}\right)=\mu_{0} H \nabla M_{0}=n K T \frac{\sinh ^{2} \xi-\xi^{2}}{\xi \sinh ^{2} \xi} \nabla \xi=\nabla\left[n K T\left(\ln \frac{\xi}{\sinh \xi}+\xi \operatorname{coth} \xi\right)\right]
$$

or

$$
\bar{h}(H)=n K T\left(\ln \frac{\xi}{\sinh \xi}+\xi \operatorname{coth} \xi\right)
$$

without loss of generality, since the arbitrary constant of the integration can be embodied inside the arbitrary constant pressure of reference $P_{0}$ from (2.10). Expression (2.14) provides us with an analytical expression for $\bar{h}(H)$. It is also easy to obtain from definition (2.6) with the aid of (2.3) a corresponding relation for $h(H)$, which is

$$
h(H)=\mu_{0} M_{0} H=\mu_{0} n m\left(\operatorname{coth} \xi-\frac{1}{\xi}\right) H=n K T(\xi \operatorname{coth} \xi-1) .
$$

Combining relationships (2.14), (2.15) and after simple analytical manipulations, we take

$$
[h(H)-\bar{h}(H)]=-n K T\left(1+\ln \frac{\xi}{\sinh \xi}\right)=-n K T \ln \frac{e \xi}{\sinh \xi},
$$

where $\xi$ contains the measure of the applied magnetic field via (2.3). Thus, the total pressure (2.10) is calculated, and, once the potentials $\Phi$ and $\Psi$ are obtained from (2.11), the flow fields (2.9) and (2.10) are known. Of course, the continuity equation (2.2) is immediately satisfied.

The basic difference of the present representation compared to that of [6] is that the equilibrium magnetization is provided by its most general expression (2.3). This difference is expressed by the additional term $\bar{h}(H)$ which appears in the pressure (2.10) and by the fact that both $h(H)$ and $\bar{h}(H)$ are calculated for the magnetization $M_{0}$, which is provided in relation (2.3). It is noted that, when $M_{0} \cong M_{s}=n m$ from the definition of $\bar{h}(H)$ in (2.10), it is obtained that $\bar{h}(H)=$ const. $\equiv 0$ (without loss of generality) and that $h(H)=h_{[6]}(H)=$ $\mu_{0} M_{s} H=\mu_{0} n m H=n K T \xi$. Consequently, representation (2.9)-(2.11) reduces to the already published general solution [6]. It is also useful to note that if the equilibrium magnetization 
$M_{0}$ is approximated by the constant value of the saturation magnetization $M_{s}$, then the last term of (2.12) becomes $h(H) \nabla\left(\ln M_{0}\right)=\mu_{0} M_{0} H \nabla\left(\ln M_{0}\right)=0$, and consequently (2.12) coincides with the momentum equation for magnetic Stokes flow presented in [6]. Finally, in order to verify the consistency of result (2.16) with the corresponding one from [6], we make use of (2.10) to rewrite the momentum equation (2.12) as follows:

$$
\eta \chi(H) \Delta \mathbf{v}=\nabla(P-[h(H)-\bar{h}(H)])=\nabla\left(P-h_{[6]}(H)\left\{\frac{[h(H)-\bar{h}(H)]}{h_{[6]}(H)}\right\}\right) .
$$

Then, we follow an easy limiting process, where with the aid of the novel result (2.16) and relation $h_{[6]}(H)=n K T \xi$ (for high values of $\xi$ ), we obtain

$$
\lim _{\xi \rightarrow+\infty} \frac{[h(H)-\bar{h}(H)]}{h_{[6]}(H)}=1
$$

which leads us to the corresponding momentum equation for magnetic Stokes flow of [6] for very high values of $\xi$, that is,

$$
\begin{aligned}
\eta \chi(H) \Delta \mathbf{v} & =\nabla\left(P-h_{[6]}(H)\right)=\nabla\left(P-\mu_{0} M_{s} H\right) \\
& =\nabla(P-n K T \xi) \quad \text { for } M_{0} \cong M_{s}=n m(\xi \longrightarrow+\infty) .
\end{aligned}
$$

In this paper we investigate the special case, where the main three-dimensional flow is perturbed by a known constant vector uniform magnetic field, arbitrarily orientated in the 3D space, of the form

$$
\mathbf{H}_{c}=\sum_{i=1}^{3} H_{c, i} \widehat{\mathbf{x}}_{i} \quad \text { with measure } H_{c}=\sqrt{\sum_{i=1}^{3} H_{c, i^{\prime}}^{2}}
$$

where this situation provides us with the constant equilibrium magnetization

$$
M_{0, c} \equiv M_{0}\left(H_{c}\right)=n m L\left(\xi_{c}\right) \quad \text { for } L\left(\xi_{c}\right)=\operatorname{coth} \xi_{c}-\frac{1}{\xi_{c}} \text { with } \xi_{c}=\frac{\mu_{0} m H_{c}}{K T}
$$

and the constant dimensionless parameter

$$
\chi_{c} \equiv \chi\left(H_{c}\right)=\left[1+\frac{\tau_{B} h_{c}}{4 \eta\left(1+\left(\tau_{S} / I\right) \tau_{B} h_{c}\right)}\right] \quad \text { for } h_{c} \equiv h\left(H_{c}\right)=\mu_{0} M_{0, c} H_{c} \text {. }
$$

Therefore, applying (2.21) and (2.22) into the magnetic Stokes equations (2.12) and (2.2), we recover the corresponding differential equations

$$
\eta \chi_{c} \Delta \mathbf{v}=\nabla\left(P-h_{c}\right)=\nabla P
$$


since $h_{c} \nabla\left(\ln M_{0, c}\right)=\nabla h_{c}=0$ and

$$
\nabla \cdot \mathbf{v}=0
$$

The particular case of the application of a constant three-dimensional magnetic field leads via relation (2.22) to $\nabla\left[\ln \chi_{c}\right]=0$ and $\nabla \bar{h}\left(H_{c}\right)=0 \Rightarrow \bar{h}\left(H_{c}\right) \equiv \bar{h}_{c}$. Therefore, our improved differential representation (2.9)-(2.11) of the solutions of (2.23) and (2.24) takes the form

$$
\begin{gathered}
\mathbf{v}=\boldsymbol{\Phi}-\frac{1}{2} \nabla(\mathbf{r} \cdot \mathbf{\Phi}+\Psi), \\
P=-\eta \chi_{c} \nabla \cdot \mathbf{\Phi}+P_{c},
\end{gathered}
$$

where $P_{c} \equiv P_{0}+\left[h_{c}-\bar{h}_{c}\right]$ specifies the new characteristic reference pressure that is arbitrarily chosen, since the total pressure enters the momentum equation (2.23) under the gradient action. As a consequence of (2.22), relations (2.11) reduce to Laplace's equations for the potentials $\boldsymbol{\Phi}$ and $\Psi$, that is,

$$
\Delta \Phi=0, \quad \Delta \Psi=0,
$$

respectively. Consequently, we have to find two harmonic potentials and calculate the magnetic flow fields from the differential representation (2.25) and (2.26) in circular cylindrical geometry, whenever the boundary conditions of the corresponding physical problem are known.

Our boundary value problem must be supplemented by the appropriate boundary conditions fixed to the precisely defined boundaries of the circular cylinder of radius $\alpha$ and of finite length $L$. Those conditions are the following:

$$
\begin{gathered}
\mathbf{v}=\mathbf{0} \quad \text { for } r=\alpha, \\
\widehat{\mathbf{r}} \cdot \mathbf{v}=\widehat{\boldsymbol{\varphi}} \cdot \mathbf{v}=0, \quad \widehat{\mathbf{z}} \cdot \mathbf{v}=v(r, \varphi) \quad \text { for } z=0, \\
\frac{\partial(\widehat{\mathbf{r}} \cdot \mathbf{v})}{\partial z}=\frac{\partial(\widehat{\boldsymbol{\varphi}} \cdot \mathbf{v})}{\partial z}=0, \quad-p+\eta \frac{\partial(\widehat{\mathbf{z}} \cdot \mathbf{v})}{\partial z}=0 \quad \text { for } z=L,
\end{gathered}
$$

where the first one defines the nonslip boundary condition on the wall of the cylinder at $r=\alpha$, the second one refers to the entrance of the cylinder at $z=0$ demanding the transversal components of the velocity to vanish and specifying a known imposed axial velocity $v(r, \varphi)$, while the third one cancels the axial derivatives of the transversal components of the velocity and demands that the axial stresses are set to zero (see also $[30,31]$ ) at the exit of the cylinder at $z=L$. Although the second part of boundary condition (2.30) comprises the thermodynamic pressure, it is to our convenience to consider small height variations, so as to neglect the hydrostatic pressure force and, consequently, to set the total pressure from (2.26) approximately equal to $p$, meaning that $P \cong p$. In addition, in order to secure consistency 
with the physical requirements of our problem, these conditions are supplemented with the demand of conservation of mass at the edge of the finite cylinder (see also [32]), that is,

$$
\nabla \cdot \mathbf{v}=0 \text { for } z=L
$$

In the limiting case, where the length of the cylinder tends to infinity, we demand that the velocity field at the outlet of the duct obtains a fully developed profile

$$
\lim _{L \rightarrow+\infty} \mathbf{v} \equiv \mathbf{v}_{\mathrm{P}}=\frac{A}{4 \eta \chi_{c}}\left(\alpha^{2}-r^{2}\right) \widehat{\mathbf{z}}, \quad \lim _{L \rightarrow+\infty} \frac{\partial P}{\partial z} \equiv \frac{d P_{\mathrm{P}}}{d z}=-A \quad \text { for } z=L,
$$

respectively, where $-A \equiv d P_{\mathrm{P}} / d z<0$ is the constant pressure gradient at the $z$-direction of the magnetic Poiseuille flow $\left(\mathbf{v}_{\mathrm{P}}, P_{\mathrm{P}}\right)$. Immediate integration gives

$$
P_{\mathrm{P}}=-A(z-L) .
$$

We point out that the constant of the integration in order to find $P_{P}$ has been taken equal to $A L$ in order to be in accordance with (2.32) and the same time keep consistency at infinity. Moreover, the effect of the magnetic field (2.20) is inherited to the velocity profile (2.32), which carries the parameter $\chi_{c}$ (in the absence of particles or magnetic field, $\chi_{c}=1$, and we obtain the hydrodynamic Poiseuille flow). On the other hand, the nature of the asymptotic condition (2.32) determines the character of the flow fields $\mathbf{v}$ and $P$. In simple words, condition (2.32) allows us to decompose the flow fields as follows:

$$
\mathbf{v}=\mathbf{v}_{\mathrm{P}}+\mathbf{v}_{g}, \quad P=P_{\mathrm{P}}+P_{g}
$$

where the pair of flow fields $\left(\mathbf{v}_{\mathrm{P}}, P_{\mathrm{P}}\right)$ refers to the Poiseuille flow, while the general velocity field $\mathbf{v}_{g}$ and the general total pressure field $P_{g}$ must satisfy the differential representation (2.25), (2.26) with (2.27) of the solutions of (2.23) and (2.24),

$$
\mathbf{v}_{g}=\mathbf{\Phi}-\frac{1}{2} \nabla(\mathbf{r} \cdot \mathbf{\Phi}+\Psi), \quad P_{g}=-\eta \chi_{c} \nabla \cdot \mathbf{\Phi}+P_{c} \quad \text { with } \Delta \mathbf{\Phi}=0, \Delta \Psi=0,
$$

in view of (2.20)-(2.22) for $\mathcal{X}_{c}$. Straightforward calculations within the frame of identities (A.3) and (A.10) confirm the satisfaction of the magnetic Stokes equations (2.23) and (2.24) by the Poiseuille flow fields $\left(\mathbf{v}_{\mathrm{P}}, P_{\mathrm{P}}\right)$ given in (2.32) and (2.33). Thus, decomposition (2.34) holds true, and the boundary condition (2.32) is substituted by

$$
\lim _{L \rightarrow+\infty} \mathbf{v}_{g}=\mathbf{0}, \quad \lim _{L \rightarrow+\infty} \frac{\partial P_{g}}{\partial z}=0 \quad \text { for } z=L,
$$

a condition that must be satisfied automatically when the 3D flow fields (2.34) are calculated.

Our goal is to solve the aforementioned boundary value problem (2.23)-(2.24) with the boundary conditions (2.28)-(2.30) supplemented with (2.31), using the decomposition (2.34) with (2.32) or (2.36), (2.33) and the differential representation (2.35) in circular cylindrical coordinates, in order to construct the three-dimensional flow fields $(\mathbf{v}, P)$ in a closed 3D analytical form, accompanied by a particular numerical implementation of the results. 


\section{The Magnetic 3D Flow Fields in Circular Cylindrical Coordinates}

According to the analysis described above, the required 3D flow fields (2.34) of our problem, in view of (2.32), (2.33), and (2.34), assume the form

$$
\mathbf{v}=u(r) \widehat{\mathbf{z}}+\mathbf{\Phi}-\frac{1}{2} \nabla(\mathbf{r} \cdot \mathbf{\Phi}+\Psi), \quad P=P_{c}+p(z)-\eta \chi_{c} \nabla \cdot \Phi,
$$

in terms of the functions ( $\chi_{c}$ provided by $(2.22)$ )

$$
u(r)=\frac{A}{4 \eta \chi_{c}}\left(\alpha^{2}-r^{2}\right), \quad p(z)=-A(z-L), \quad \text { where } A=\frac{-d p(z)}{d z}=\text { const. }>0 .
$$

Since our case involves an interior flow problem, it is imposed the use of regular solutions on the axis of symmetry of the circular cylinder $(r=0)$, which means that the Neumann functions $N_{n}(\mu r)$ must be excluded from any general harmonic expansion of the type (A.30). The constant parameter $\mu \in \mathbb{R}$ from the separation of variables of the Laplace equation in our system (see the appendix for that matter) will be determined from boundary condition (2.28) in the end of our analytical procedure. However, until then, we will introduce the symbol " $\int \Sigma_{\mu} \ldots$ ", which denotes integration if $\mu$ takes continuous values or summation in the case where $\mu$ is a parameter with discrete values. Consequently, the complete representation of the regular potentials $\Phi$ and $\Psi$, which belong to the kernel space of $\Delta$, is

$$
\mathbf{\Phi}=\sum_{n=0}^{\infty} \int \sum_{\mu} \sum_{i=1}^{4} \mathbf{e}_{n}^{\mu,(i)} H_{n}^{\mu,(i)}, \quad \Psi=\sum_{n=0}^{\infty} \int \sum_{\mu} \sum_{i=1}^{4} d_{n}^{\mu,(i)} H_{n}^{\mu,(i)}
$$

where, for $n \geq 0, \mu \in \mathbb{R}$, and $i=1,2,3,4$, the coefficients $\mathbf{e}_{n}^{\mu,(i)}=a_{n}^{\mu,(i)} \widehat{\mathbf{x}}_{1}+b_{n}^{\mu,(i)} \widehat{\mathbf{x}}_{2}+c_{n}^{\mu,(i)} \widehat{\mathbf{x}}_{3}$ and $d_{n}^{\mu,(i)}$ denote the unknown vector and the scalar constant coefficients of the harmonic potentials $\Phi$ and $\Psi$, respectively. On the other hand, within the frame of (A.30) the $\mathrm{H}$ functions, usually named as eigenfunctions, for every kind $i=1,2,3,4$ are

$$
\begin{array}{ll}
H_{n}^{\mu,(1)}=J_{n}(\mu r) \sin n \varphi \cosh (\mu z) & \text { for } \mu \in \mathbb{R}, \\
H_{n}^{\mu,(2)}=J_{n}(\mu r) \sin n \varphi \sinh (\mu z) & \text { for } \mu \in \mathbb{R}, \\
H_{n}^{\mu,(3)}=J_{n}(\mu r) \cos n \varphi \cosh (\mu z) & \text { for } \mu \in \mathbb{R}, \\
H_{n}^{\mu,(4)}=J_{n}(\mu r) \cos n \varphi \sinh (\mu z) & \text { for } \mu \in \mathbb{R},
\end{array}
$$

where $n \geq 0$, while all the needed information for the Bessel, the trigonometric, and the hyperbolic functions is summarized in the appendix. Inserting the potentials (3.3) in the flow fields (3.1) and by extensive use of identities (A.2), (A.3), and (A.5), we derive the relation

$$
\mathbf{v}=u(r) \widehat{\mathbf{z}}+\frac{1}{2} \sum_{n=0}^{\infty} \int \sum_{\mu} \sum_{i=1}^{4}\left\{\mathbf{e}_{n}^{\mu_{,}(i)} H_{n}^{\mu,(i)}-\left[\left(\mathbf{e}_{n}^{\mu_{,}(i)} \cdot \mathbf{r}\right)+d_{n}^{\mu,(i)}\right] \nabla H_{n}^{\mu,(i)}\right\}
$$


for the velocity field, while for the total pressure field we obtain

$$
P=P_{c}+p(z)-\eta X_{c} \sum_{n=0}^{\infty} \int \sum_{\mu} \sum_{i=1}^{4}\left\{\mathbf{e}_{n}^{\mu,(i)} \cdot \nabla H_{n}^{\mu,(i)}\right\}
$$

in the cylinder's prescribed dimensions $V\left(\mathbb{R}^{3}\right)$. That way, the generality of the potentials is inherited to the magnetic flow fields (3.8) and (3.9), via the set of unknown constant coefficients $\mathbf{e}_{n}^{\mu,(i)}$ and $d_{n}^{\mu,(i)}$ for $n \geq 0, \mu \in \mathbb{R}$, and $i=1,2,3,4$, which have to be calculated explicitly from the proper conditions (2.28)-(2.31). Additionally, the parameter $\mu$ will be evaluated from the same conditions.

Since the vector character of the vector harmonic potential $\mathbf{\Phi}$ is reflected upon the corresponding constant coefficients, which are written in Cartesian coordinates, we are obliged to work in the Cartesian system. Thereupon, before we proceed to the boundary conditions, it is necessary, for our convenience in calculations, to evaluate the gradient of the harmonic $H$-functions (3.4)-(3.7) that appear in the flow fields (3.8) and (3.9). Obviously $\Delta\left(\nabla H_{n}^{\mu,(i)}\right)=\nabla\left(\Delta H_{n}^{\mu,(i)}\right)=0, n \geq 0, \mu \in \mathbb{R}, i=1,2,3,4$, which means that $\nabla H_{n}^{\mu,(i)}$ belong to the subspace produced by $H_{n}^{\mu,(i)}$, and it is feasible to be written as a function of them in Cartesian coordinates. Therefore, we will work on Cartesian coordinates as far as the vector character of the velocity (3.8) is concerned, and using the transformation (A.14) we will return to the circular cylindrical basis $\widehat{\mathbf{r}}, \widehat{\boldsymbol{\varphi}}, \widehat{\mathbf{z}}$ (see also (A.13)). Hence, in view of (A.13), (A.15) and the basic relations for the trigonometric functions (A.25)-(A.28), utilizing the recurrence relations for the Bessel functions (A.22), (A.23), and in terms of the $H$-functions (3.4)-(3.7), for $i=1$, we are led to

$$
\begin{aligned}
\nabla H_{n}^{\mu,(1)}= & \left(\widehat{\mathbf{r}} \frac{\partial}{\partial r}+\frac{\widehat{\boldsymbol{\varphi}}}{r} \frac{\partial}{\partial \varphi}+\widehat{\mathbf{z}} \frac{\partial}{\partial z}\right)\left[J_{n}(\mu r) \sin n \varphi \cosh (\mu z)\right] \\
= & \widehat{\mathbf{r}} \mu \frac{d\left(J_{n}(\mu r)\right)}{d(\mu r)} \sin n \varphi \cosh (\mu z)+\frac{\widehat{\varphi}}{\mu r} \mu n J_{n}(\mu r) \cos n \varphi \cosh (\mu z) \\
& +\widehat{\mathbf{z}} \mu J_{n}(\mu r) \sin n \varphi \sinh (\mu z) \\
= & \mu J_{n}(\mu r) \sin n \varphi \sinh (\mu z) \widehat{\mathbf{x}}_{1}+\mu \cosh (\mu z) \\
& \times\left[\widehat{\mathbf{x}}_{2}\left(\cos \varphi \sin n \varphi J_{n}^{\prime}(\mu r)-\sin \varphi \cos n \varphi \frac{n}{\mu r} J_{n}(\mu r)\right)\right. \\
& \left.\quad+\widehat{\mathbf{x}}_{3}\left(\sin \varphi \sin n \varphi J_{n}^{\prime}(\mu r)+\cos \varphi \cos n \varphi \frac{n}{\mu r} J_{n}(\mu r)\right)\right] \\
= & \mu H_{n}^{\mu,(2)} \widehat{\mathbf{x}}_{1}+\frac{1}{2} \mu \cosh (\mu z)\left[\widehat{\mathbf{x}}_{2}\left(-J_{n+1}(\mu r) \sin (n+1) \varphi+J_{n-1}(\mu r) \sin (n-1) \varphi\right)\right. \\
\quad & \left.+\widehat{\mathbf{x}}_{3}\left(J_{n-1}(\mu r) \cos (n-1) \varphi+J_{n+1}(\mu r) \cos (n+1) \varphi\right)\right],
\end{aligned}
$$

or

$$
\nabla H_{n}^{\mu,(1)}=\mu\left[H_{n}^{\mu,(2)} \widehat{\mathbf{x}}_{1}+\frac{1}{2}\left(-H_{n+1}^{\mu,(1)}+H_{n-1}^{\mu,(1)}\right) \widehat{\mathbf{x}}_{2}+\frac{1}{2}\left(H_{n+1}^{\mu,(3)}+H_{n-1}^{\mu,(3)}\right) \widehat{\mathbf{x}}_{3}\right],
$$


for every $n \geq 0$ and $\mu \in \mathbb{R}$, where the prime at the Bessel functions denotes derivation with respect to the variable $\mu r \in \mathbb{R}$. In the same way we calculate the rest three gradients, that is,

$$
\begin{aligned}
& \nabla H_{n}^{\mu,(2)}=\mu\left[H_{n}^{\mu,(1)} \widehat{\mathbf{x}}_{1}+\frac{1}{2}\left(-H_{n+1}^{\mu,(2)}+H_{n-1}^{\mu,(2)}\right) \widehat{\mathbf{x}}_{2}+\frac{1}{2}\left(H_{n+1}^{\mu,(4)}+H_{n-1}^{\mu,(4)}\right) \widehat{\mathbf{x}}_{3}\right], \\
& \nabla H_{n}^{\mu,(3)}=\mu\left[H_{n}^{\mu,(4)} \widehat{\mathbf{x}}_{1}+\frac{1}{2}\left(-H_{n+1}^{\mu,(3)}+H_{n-1}^{\mu,(3)}\right) \widehat{\mathbf{x}}_{2}-\frac{1}{2}\left(H_{n+1}^{\mu,(1)}+H_{n-1}^{\mu,(1)}\right) \widehat{\mathbf{x}}_{3}\right], \\
& \nabla H_{n}^{\mu,(4)}=\mu\left[H_{n}^{\mu,(3)} \widehat{\mathbf{x}}_{1}+\frac{1}{2}\left(-H_{n+1}^{\mu,(4)}+H_{n-1}^{\mu,(4)}\right) \widehat{\mathbf{x}}_{2}-\frac{1}{2}\left(H_{n+1}^{\mu,(2)}+H_{n-1}^{\mu,(2)}\right) \widehat{\mathbf{x}}_{3}\right],
\end{aligned}
$$

for every $n \geq 0$ and $\mu \in \mathbb{R}$, where it is obvious that $\Delta\left(\nabla H_{n}^{\mu,(i)}\right)=\nabla\left(\Delta H_{n}^{\mu,(i)}\right)=0$. Here, we must add that, in order to avoid negative values of $n$ within the above relationships, we impose

$$
H_{-n}^{\mu,(i)} \equiv 0, \quad n \geq 0, \mu \in \mathbb{R}, i=1,2,3,4
$$

Expressions (3.11) and (3.12) can be collected in the general formulae

$$
\nabla H_{n}^{\mu,(i)}=\mu\left[H_{n}^{\mu,\left(a_{i}\right)} \widehat{\mathbf{x}}_{1}+\frac{1}{2}\left(-H_{n+1}^{\mu,(i)}+H_{n-1}^{\mu,(i)}\right) \widehat{\mathbf{x}}_{2}+\frac{c_{i}}{2}\left(H_{n+1}^{\mu,\left(b_{i}\right)}+H_{n-1}^{\mu,\left(b_{i}\right)}\right) \widehat{\mathbf{x}}_{3}\right], \quad \mu \in \mathbb{R},
$$

for every $n \geq 0$, while $a_{i}=i+(-1)^{i+1}, b_{i}=i+2 c_{i}$ for $i=1,2,3,4$ and $c_{i}=1, i=1,2$ or $c_{i}=-1, i=3,4$. Relation (A.12) and Cartesian definition of $\mathbf{e}_{n}^{\mu,(i)}$ result in the product

$$
\left(\mathbf{e}_{n}^{\mu_{,}(i)} \cdot \mathbf{r}\right)=a_{n}^{\mu,(i)} z+b_{n}^{\mu,(i)} r \cos \varphi+c_{n}^{\mu,(i)} r \sin \varphi, \quad n \geq 0, \mu \in \mathbb{R}, i=1,2,3,4,
$$

which by virtue of (3.14) incorporates with (3.8) to the following velocity field:

$$
\begin{aligned}
\mathbf{v}= & u(r) \widehat{\mathbf{z}} \\
+\frac{1}{2} \sum_{n=0}^{\infty} \int \sum_{\mu} \sum_{i=1}^{4}\{ & \left(a_{n}^{\mu,(i)} \widehat{\mathbf{x}}_{1}+b_{n}^{\mu,(i)} \widehat{\mathbf{x}}_{2}+c_{n}^{\mu,(i)} \widehat{\mathbf{x}}_{3}\right) H_{n}^{\mu,(i)} \\
& -\mu\left(a_{n}^{\mu,(i)} z+b_{n}^{\mu,(i)} r \cos \varphi+c_{n}^{\mu,(i)} r \sin \varphi+d_{n}^{\mu,(i)}\right) \\
& \left.\times\left[H_{n}^{\mu,\left(a_{i}\right)} \widehat{\mathbf{x}}_{1}+\frac{1}{2}\left(-H_{n+1}^{\mu,(i)}+H_{n-1}^{\mu,(i)}\right) \widehat{\mathbf{x}}_{2}+\frac{c_{i}}{2}\left(H_{n+1}^{\mu,\left(b_{i}\right)}+H_{n-1}^{\mu,\left(b_{i}\right)}\right) \widehat{\mathbf{x}}_{3}\right]\right\} .
\end{aligned}
$$


At this point we manipulate properly the $H$-functions $H_{n+1}^{\mu,(i)}, H_{n-1}^{\mu,(i)}, H_{n+1}^{\mu,\left(b_{i}\right)}$, and $H_{n-1}^{\mu,\left(b_{i}\right)}$ by a certain readjustment of the index $n$ at the series to rewrite the velocity (3.16) as

$$
\begin{array}{r}
\mathbf{v}=u(r) \widehat{\mathbf{z}} \\
+\frac{1}{2} \sum_{n=0}^{\infty} \int \sum_{\mu} \sum_{i=1}^{4}\left\{\widehat{\mathbf{x}}_{1}\left[a_{n}^{\mu,(i)} H_{n}^{\mu,(i)}-\mu\left(a_{n}^{\mu,(i)} z+b_{n}^{\mu,(i)} r \cos \varphi+c_{n}^{\mu,(i)} r \sin \varphi+d_{n}^{\mu,(i)}\right) H_{n}^{\mu,\left(a_{i}\right)}\right]\right. \\
+\widehat{\mathbf{x}}_{2}\left[b_{n}^{\mu,(i)} H_{n}^{\mu,(i)}+\left(A_{n,-}^{\mu,(i)} z+B_{n,-}^{\mu,(i)} r \cos \varphi+C_{n,-}^{\mu,(i)} r \sin \varphi+D_{n^{-}}^{\mu,(i)}\right) H_{n}^{\mu,(i)}\right] \\
\left.+\widehat{\mathbf{x}}_{3}\left[c_{n}^{\mu,(i)} H_{n}^{\mu,(i)}-c_{i}\left(A_{n,+}^{\mu,(i)} z+B_{n,+}^{\mu,(i)} r \cos \varphi+C_{n,+}^{\mu,(i)} r \sin \varphi+D_{n,+}^{\mu,(i)}\right) H_{n}^{\mu,\left(b_{i}\right)}\right]\right\},
\end{array}
$$

where we note that $\widehat{\mathbf{z}}=\widehat{\mathbf{x}}_{1}$, the parabolic velocity profile $u(r)$ is given by (3.2), and the new constant coefficients into the velocity field (3.17) are provided as a function of $a_{n}^{\mu,(i)}, b_{n}^{\mu,(i)}$, $c_{n}^{\mu,(i)}$, and $d_{n}^{\mu,(i)}$ for $n \geq 0, \mu \in \mathbb{R}$, and $i=1,2,3,4$, which are grouped via the simple relations

$$
\begin{aligned}
& A_{n, \pm}^{\mu,(i)}=\frac{\mu}{2}\left(a_{n-1}^{\mu,(i)} \pm a_{n+1}^{\mu,(i)}\right) \quad \text { with } a_{-1}^{\mu,(i)} \equiv 0 \text { for } n \geq 0, \mu \in \mathbb{R}, i=1,2,3,4, \\
& B_{n, \pm}^{\mu,(i)}=\frac{\mu}{2}\left(b_{n-1}^{\mu,(i)} \pm b_{n+1}^{\mu,(i)}\right) \quad \text { with } b_{-1}^{\mu,(i)} \equiv 0 \text { for } n \geq 0, \mu \in \mathbb{R}, i=1,2,3,4, \\
& C_{n, \pm}^{\mu,(i)}=\frac{\mu}{2}\left(c_{n-1}^{\mu,(i)} \pm c_{n+1}^{\mu,(i)}\right) \quad \text { with } c_{-1}^{\mu,(i)} \equiv 0 \text { for } n \geq 0, \mu \in \mathbb{R}, i=1,2,3,4, \\
& D_{n, \pm}^{\mu,(i)}=\frac{\mu}{2}\left(d_{n-1}^{\mu,(i)} \pm d_{n+1}^{\mu,(i)}\right) \quad \text { with } d_{-1}^{\mu,(i)} \equiv 0 \text { for } n \geq 0, \mu \in \mathbb{R}, i=1,2,3,4 .
\end{aligned}
$$

Similarly, formulae (3.14) and the group of constant coefficients (3.18)-(3.21) are interrelated, and the total pressure (3.9) becomes

$$
\begin{aligned}
& P=P_{c}+p(z)-\eta X_{c} \sum_{n=0}^{\infty} \int \sum_{\mu} \sum_{i=1}^{4}\{ \mu\left(a_{n}^{\mu,(i)} \widehat{\mathbf{x}}_{1}+b_{n}^{\mu,(i)} \widehat{\mathbf{x}}_{2}+c_{n}^{\mu,(i)} \widehat{\mathbf{x}}_{3}\right) \\
&\left.\cdot\left[H_{n}^{\mu,\left(a_{i}\right)} \widehat{\mathbf{x}}_{1}+\frac{1}{2}\left(-H_{n+1}^{\mu,(i)}+H_{n-1}^{\mu,(i)}\right) \widehat{\mathbf{x}}_{2}+\frac{c_{i}}{2}\left(H_{n+1}^{\mu,\left(b_{i}\right)}+H_{n-1}^{\mu,\left(b_{i}\right)}\right) \widehat{\mathbf{x}}_{3}\right]\right\},
\end{aligned}
$$

or since $\widehat{\mathbf{x}}_{i} \cdot \widehat{\mathbf{x}}_{j}=\delta_{i j}\left(\delta_{i j}\right.$ being the Kronecker delta) and with a proper readjustment of the index $n$ at the series for the $H$-functions $H_{n+1}^{\mu,(i)}, H_{n-1}^{\mu,(i)}, H_{n+1}^{\mu,\left(b_{i}\right)}$, and $H_{n-1}^{\mu,\left(b_{i}\right)}$ as previously mentioned, relation (3.22) yields

$$
P=P_{c}+p(z)-\eta X_{c} \sum_{n=0}^{\infty} \int \sum_{\mu} \sum_{i=1}^{4}\left[\mu a_{n}^{\mu,(i)} H_{n}^{\mu,\left(a_{i}\right)}-B_{n,-}^{\mu,(i)} H_{n}^{\mu,(i)}+c_{i} C_{n,+}^{\mu,(i)} H_{n}^{\mu,\left(b_{i}\right)}\right],
$$


for the total pressure field, where the corresponding pressure $p(z)$ of the parabolic velocity profile is provided by (3.2). The velocity (3.17) and the total pressure (3.23) of the micropolar fluid are ready to accept the boundary conditions (2.28)-(2.30) and condition (2.31) of our physical problem, in order to determine the unknown constant coefficients $a_{n}^{\mu,(i)}, b_{n}^{\mu,(i)}, c_{n}^{\mu,(i)}$, and $d_{n}^{\mu_{1}(i)}$ for $n \geq 0$ and $i=1,2,3,4$, as well as the parameter $\mu \in \mathbb{R}$. During this process, we must keep in mind that $a_{i}=i+(-1)^{i+1}, b_{i}=i+2 c_{i}$ for $i=1,2,3,4$ and $c_{i}=1, i=1,2$ or $c_{i}=-1, i=3,4$.

We begin with the first part of the inlet boundary condition (2.29) at the entrance of the circular cylinder $z=0$. Applying this condition on the velocity field (3.17), condition $\widehat{\mathbf{r}} \cdot \mathbf{v}=0$ at $z=0$ results in

$$
\begin{aligned}
\sum_{n=0}^{\infty} \int \sum_{\mu} \sum_{i=1,3}\left\{\left(\widehat{\mathbf{r}} \cdot \widehat{\mathbf{x}}_{2}\right)\right. & {\left[b_{n}^{\mu,(i)} H_{n}^{\mu,(i)}+\left(B_{n,-}^{\mu,(i)} r \cos \varphi+C_{n,-}^{\mu,(i)} r \sin \varphi+D_{n,-}^{\mu,(i)}\right) H_{n}^{\mu,(i)}\right] } \\
& \left.+\left(\widehat{\mathbf{r}} \cdot \widehat{\mathbf{x}}_{3}\right)\left[c_{n}^{\mu,(i)} H_{n}^{\mu,(i)}-c_{i}\left(B_{n,+}^{\mu,(i)} r \cos \varphi+C_{n,+}^{\mu,(i)} r \sin \varphi+D_{n,+}^{\mu,(i)}\right) H_{n}^{\mu,\left(b_{i}\right)}\right]\right\}=0, \quad z=0,
\end{aligned}
$$

since $\widehat{\mathbf{r}} \cdot \widehat{\mathbf{z}}=\widehat{\mathbf{r}} \cdot \widehat{\mathbf{x}}_{1}=0$, while $\widehat{\boldsymbol{\varphi}} \cdot \mathbf{v}=0$ at $z=0$ renders

$$
\begin{aligned}
\sum_{n=0}^{\infty} \int \sum_{\mu} \sum_{i=1,3}\{( & \left(\widehat{\boldsymbol{\varphi}} \cdot \widehat{\mathbf{x}}_{2}\right)\left[b_{n}^{\mu,(i)} H_{n}^{\mu,(i)}+\left(B_{n,-}^{\mu,(i)} r \cos \varphi+C_{n,-}^{\mu,(i)} r \sin \varphi+D_{n,-}^{\mu,(i)}\right) H_{n}^{\mu,(i)}\right] \\
& \left.+\left(\widehat{\boldsymbol{\varphi}} \cdot \widehat{\mathbf{x}}_{3}\right)\left[c_{n}^{\mu,(i)} H_{n}^{\mu,(i)}-c_{i}\left(B_{n,+}^{\mu,(i)} r \cos \varphi+C_{n,+}^{\mu,(i)} r \sin \varphi+D_{n,+}^{\mu,(i)}\right) H_{n}^{\mu,\left(b_{i}\right)}\right]\right\}=0, \quad z=0,
\end{aligned}
$$

since $\hat{\boldsymbol{\varphi}} \cdot \widehat{\mathbf{z}}=\hat{\boldsymbol{\varphi}} \cdot \widehat{\mathbf{x}}_{1}=0$. We notice that both conditions (3.24) and (3.25) contain the $H$ eigenfunctions of kind $i=1,3$ (relations (3.4), (3.6)), where those survive for $z=0$ as it is revealed from (A.29). Hence, we utilize definitions (3.4) and (3.6), relations (A.13) or (A.14), as well as (3.19)-(3.21), in order to handle firstly condition (3.24) with extensive use of the recurrence relations of the trigonometric functions (A.25)-(A.28). Therefore, after long and tedious calculations on (3.24) with orthogonality arguments of the trigonometric functions $\sin n \varphi, n \geq 1$, and via the definition for the Bessel functions (A.19), we obtain the following relations for the constant coefficients:

$$
\begin{aligned}
& \mu d_{n}^{\mu,(1)}+(n+2)\left(b_{n+1}^{\mu,(1)}-c_{n+1}^{\mu,(3)}\right)=0 \text { for } n \geq 1, \mu \in \mathbb{R}, \\
& \mu d_{n}^{\mu,(1)}+(n-2)\left(b_{n-1}^{\mu,(1)}+c_{n-1}^{\mu,(3)}\right)=0 \text { for } n \geq 1, \mu \in \mathbb{R}, \\
& \mu d_{n}^{\mu,(1)}+(n-2)\left(b_{n+1}^{\mu,(1)}-c_{n+1}^{\mu,(3)}\right)=0 \quad \text { for } n \geq 1, \mu \in \mathbb{R},
\end{aligned}
$$

while for $n=0$ we can admit without loss of generality $(\sin 0 \varphi=0)$ that $d_{0}^{\mu,(1)}=b_{0}^{\mu,(1)}=c_{0}^{\mu,(3)}=$ $0, \mu \in \mathbb{R}$. On the other hand, an easy manipulation of (3.26) reveals the nihilism of the constant coefficients $d_{n}^{\mu,(1)}, b_{n}^{\mu,(1)}$, and $c_{n}^{\mu,(3)}$ for $n \geq 1$ and $\mu \in \mathbb{R}$. Recapitulating,

$$
d_{n}^{\mu,(1)}=b_{n}^{\mu_{,}(1)}=c_{n}^{\mu_{,}(3)}=0 \quad \text { for } n \geq 0, \mu \in \mathbb{R},
$$


whilst orthogonality of $\cos n \varphi, n \geq 0$, on (3.24) is provided with similar relations to (3.26), leading to

$$
d_{n}^{\mu,(3)}=b_{n}^{\mu,(3)}=c_{n}^{\mu,(1)}=0 \quad \text { for } n \geq 0, \mu \in \mathbb{R} .
$$

Since we have finished with condition (3.24), we deal, now, with condition (3.25), and we observe that results (3.27), (3.28) correlate with (3.18)-(3.21) so as to satisfy automatically this condition (3.25). Hence, we completed our analysis with the first part of the inlet boundary condition (2.29), which implied (see (3.27) and (3.28))

$$
d_{n}^{\mu,(i)}=b_{n}^{\mu,(i)}=c_{n}^{\mu,(i)}=0 \quad \text { for } n \geq 0, \mu \in \mathbb{R}, i=1,3,
$$

and via (3.18)-(3.21)

$$
D_{n, \pm}^{\mu,(i)}=B_{n, \pm}^{\mu,(i)}=C_{n, \pm}^{\mu,(i)}=0 \quad \text { for } n \geq 0, \mu \in \mathbb{R}, i=1,3 .
$$

It is worth mentioning that the very same results are obtained in an easier way by using the velocity field from (3.8) and not from (3.17). Nevertheless, it was a worthwhile way to crosscheck the relations (3.29).

At this point, before we proceed to the next boundary conditions, we will write down the flow fields with their form until now. Under this aim, we insert relationships (3.29), (3.30) into the flow fields (3.17) and (3.23), taking into account the constant parameters $a_{i}, b_{i}, c_{i}$ for $i=1,2,3$, 4 with the definitions for the $H$-functions (3.4)-(3.7), and we recover the velocity field

$$
\begin{gathered}
\mathbf{v}=u(r) \widehat{\mathbf{z}}+\frac{\widehat{\mathbf{x}}_{1}}{2} \sum_{n=0}^{\infty} \int \sum_{\mu} J_{n}(\mu r)\left\{\left[\left(a_{n}^{\mu,(1)}-\mu\left(a_{n}^{\mu,(2)} z+b_{n}^{\mu,(2)} r \cos \varphi+c_{n}^{\mu,(2)} r \sin \varphi+d_{n}^{\mu,(2)}\right)\right) \sin n \varphi\right.\right. \\
\left.+\left(a_{n}^{\mu,(3)}-\mu\left(a_{n}^{\mu,(4)} z+b_{n}^{\mu,(4)} r \cos \varphi+c_{n}^{\mu,(4)} r \sin \varphi+d_{n}^{\mu,(4)}\right)\right) \cos n \varphi\right] \\
\times \cosh (\mu z)+\left[\left(a_{n}^{\mu,(2)}-a_{n}^{\mu,(1)} \mu z\right) \sin n \varphi\right. \\
\left.\left.+\left(a_{n}^{\mu,(4)}-a_{n}^{\mu,(3)} \mu z\right) \cos n \varphi\right] \sinh (\mu z)\right\} \\
+\frac{\widehat{\mathbf{x}}_{2}}{2} \sum_{n=0}^{\infty} \int \sum_{\mu} J_{n}(\mu r)\left\{\left[\left(b_{n}^{\mu,(2)}+A_{n,-}^{\mu,(2)} z+B_{n,-}^{\mu,(2)} r \cos \varphi+C_{n,-}^{\mu,(2)} r \sin \varphi+D_{n,-}^{\mu,(2)}\right) \sin n \varphi\right.\right. \\
\left.+\left(b_{n}^{\mu,(4)}+A_{n,-}^{\mu,(4)} z+B_{n,-}^{\mu,(4)} r \cos \varphi+C_{n,-}^{\mu,(4)} r \sin \varphi+D_{n,-}^{\mu,(4)}\right) \cos n \varphi\right] \\
\left.\times \sinh (\mu z)+\left[A_{n,-}^{\mu,(1)} \sin n \varphi+A_{n,-}^{\mu,(3)} \cos n \varphi\right] z \cosh (\mu z)\right\} \\
+\frac{\widehat{\mathbf{x}}_{3}}{2} \sum_{n=0}^{\infty} \int \sum_{\mu} J_{n}(\mu r)\left\{\left[\left(c_{n}^{\mu,(2)}+A_{n,+}^{\mu,(4)} z+B_{n,+}^{\mu,(4)} r \cos \varphi+C_{n,+}^{\mu,(4)} r \sin \varphi+D_{n,+}^{\mu,(4)}\right) \sin n \varphi\right.\right. \\
\left.+\left(c_{n}^{\mu,(4)}-A_{n,+}^{\mu,(2)} z-B_{n,+}^{\mu,(2)} r \cos \varphi-C_{n,+}^{\mu,(2)} r \sin \varphi-D_{n,+}^{\mu,(2)}\right) \cos n \varphi\right] \\
\times \\
\left.\sinh (\mu z)+\left[A_{n,+}^{\mu,(3)} \sin n \varphi-A_{n,+}^{\mu,(1)} \cos n \varphi\right] z \cosh (\mu z)\right\},
\end{gathered}
$$


while for the total pressure field we arrive at

$$
\begin{aligned}
P=P_{c}+p(z)-\eta \chi_{c} \sum_{n=0}^{\infty} \int \sum_{\mu} J_{n}(\mu r)\{[ & \left.\mu\left(a_{n}^{\mu,(2)} \sin n \varphi+a_{n}^{\mu,(4)} \cos n \varphi\right)\right] \cosh (\mu z) \\
+ & {\left[\left(\mu a_{n}^{\mu,(1)}-B_{n,-}^{\mu,(2)}-C_{n,+}^{\mu,(4)}\right) \sin n \varphi\right.} \\
& \left.\left.+\left(\mu a_{n}^{\mu,(3)}-B_{n,-}^{\mu,(4)}+C_{n,+}^{\mu,(2)}\right) \cos n \varphi\right] \sinh (\mu z)\right\},
\end{aligned}
$$

in terms of the constant coefficients (3.18)-(3.21) and the factor (2.22), where $u(r), p(z)$ are given via relation (3.2).

Next, we refer to the first part of the outlet boundary condition (2.30), which forces us to set the axial derivatives of the transversal components of the velocity to nil. Since the velocity field (3.31) is written in Cartesian coordinates with $\widehat{\mathbf{z}}=\widehat{\mathbf{x}}_{1}$, we replace this part of (2.30) by the proper identical relationships

$$
\frac{\partial(\widehat{\mathbf{r}} \cdot \mathbf{v})}{\partial z}=\frac{\partial(\widehat{\boldsymbol{\varphi}} \cdot \mathbf{v})}{\partial z}=0 \Longrightarrow \frac{\partial\left(\widehat{\mathbf{x}}_{2} \cdot \mathbf{v}\right)}{\partial z}=\frac{\partial\left(\widehat{\mathbf{x}}_{3} \cdot \mathbf{v}\right)}{\partial z}=0 \quad \text { for } z=L,
$$

where we have used the fact that $\partial \widehat{\mathbf{r}} / \partial z=\partial \widehat{\boldsymbol{\varphi}} / \partial z=\mathbf{0}$ (see (A.13)), while for reasons of convenience to our forthcoming manipulations we incorporate the two relations of (3.33) into

$$
\frac{\partial\left(\mathbf{v} \cdot \widehat{\mathbf{x}}_{(2)}\right)}{\partial z}=0 \quad \text { for } z=L .
$$

For the respective calculations based on (3.34), the upper index will refer to $\widehat{\mathbf{x}}_{2}$, whilst the lower one will correspond to $\widehat{\mathbf{x}}_{3}$. Within this consideration, we apply the boundary conditions (3.34) to the velocity field (3.31), and since $\widehat{\mathbf{z}} \cdot \widehat{\mathbf{x}}_{2}=\widehat{\mathbf{z}} \cdot \widehat{\mathbf{x}}_{3}=0$ with $\widehat{\mathbf{z}}=\widehat{\mathbf{x}}_{1}$, this act gives rise to

$$
\begin{aligned}
& \sum_{n=0}^{\infty} \int \sum_{\mu}\left\{\mu ( \frac { J _ { n } ( \mu r ) } { \mu r } ) \left[\operatorname { s i n } n \varphi \left(A_{n, \mp}^{\mu,\left(\begin{array}{l}
1 \\
3
\end{array}\right)}(\cosh (\mu L)+\mu L \sinh (\mu L))\right.\right.\right. \\
& +A_{n, \mp}^{\mu,\left(\begin{array}{l}
2 \\
4
\end{array}\right)}(\sinh (\mu L)+\mu L \cosh (\mu L)) \\
& \left.+\left(\begin{array}{l}
b \\
c
\end{array}\right)_{n}^{\mu,(2)} \mu \cosh (\mu L)+D_{n, \mp}^{\mu,\left(\begin{array}{l}
2 \\
4
\end{array}\right)} \mu \cosh (\mu L)\right) \\
& +\cos n \varphi\left( \pm A_{n, \mp}^{\mu,\left(\begin{array}{l}
3 \\
1
\end{array}\right)}(\cosh (\mu L)+\mu L \sinh (\mu L))\right. \\
& \pm A_{n, \mp}^{\mu,\left(\begin{array}{l}
4 \\
2
\end{array}\right)}(\sinh (\mu L)+\mu L \cosh (\mu L)) \\
& \left.\left.+\left(\begin{array}{l}
b \\
c
\end{array}\right)_{n}^{\mu,(4)} \mu \cosh (\mu L) \pm D_{n, \mp}^{\mu,\left(\begin{array}{l}
4 \\
2
\end{array}\right)} \mu \cosh (\mu L)\right)\right] \\
& +\mu \cosh (\mu L) J_{n}(\mu r)\left[B_{n, \mp}^{\mu,(2} \begin{array}{c}
2 \\
4
\end{array}\right) \cos \varphi \sin n \varphi+C_{n, \mp}^{\mu,\left(\begin{array}{l}
2 \\
4
\end{array}\right)} \sin \varphi \sin n \varphi \\
& \left.\left. \pm B_{n, \mp}^{\mu,\left(\begin{array}{l}
4 \\
2
\end{array}\right)} \cos \varphi \cos n \varphi \pm C_{n, \mp}^{\mu,\left(\begin{array}{l}
4 \\
2
\end{array}\right)} \sin \varphi \cos n \varphi\right]\right\}=0,
\end{aligned}
$$


which are two similar relations at $z=L$, and the case $n=0$ is taken by the general case for $n \geq 1$ into (3.35). We take into account the recurrence relations of the trigonometric functions (A.25)-(A.28), we use the Bessel recurrence relation (A.23), and, after some calculations via (A.19), orthogonality of $\sin n \varphi, n \geq 0$ and a certain readjustment of the index $n$ at the series infer the following formulae:

$$
\begin{aligned}
A_{n, \mp}^{\mu,\left(\begin{array}{l}
1 \\
3
\end{array}\right)}(\cosh (\mu L)+\mu L \sinh (\mu L))+A_{n, \mp}^{\mu,\left(\begin{array}{l}
2 \\
4
\end{array}\right)}(\sinh (\mu L)+\mu L \cosh (\mu L)) \\
\quad+\left[\left(\begin{array}{l}
b \\
c
\end{array}\right)_{n}^{\mu,(2)}+D_{n, \mp}^{\left.\mu,\left(\begin{array}{l}
2 \\
4
\end{array}\right)\right] \mu \cosh (\mu L)}\right. \\
\quad=-n \cosh (\mu L)\left(B_{n-1, \mp}^{\mu,\left(\begin{array}{l}
2 \\
4
\end{array}\right)} \pm C_{n-1, \mp}^{\mu,\left(\begin{array}{l}
4 \\
2
\end{array}\right)}\right)=-n \cosh (\mu L)\left(B_{n+1, \mp}^{\mu,\left(\begin{array}{l}
2 \\
4
\end{array}\right)} \mp C_{n+1, \mp}^{\left.\mu,\left(\begin{array}{l}
4 \\
2
\end{array}\right)\right)} \text { for } n \geq 0, \mu \in \mathbb{R} .\right.
\end{aligned}
$$

Orthogonality arguments on the functions $\cos n \varphi, n \geq 0$ with similar readjustment of $n$ imply

$$
\begin{aligned}
& \pm A_{n, \mp}^{\mu,\left(\begin{array}{l}
3 \\
1
\end{array}\right)}(\cosh (\mu L)+\mu L \sinh (\mu L)) \pm A_{n, \mp}^{\mu,\left(\begin{array}{l}
4 \\
2
\end{array}\right)}(\sinh (\mu L)+\mu L \cosh (\mu L)) \\
& \quad+\left[\left(\begin{array}{l}
b \\
c
\end{array}\right)_{n}^{\mu,(4)} \pm D_{n, \mp}^{\mu,\left(\begin{array}{l}
4 \\
2
\end{array}\right)}\right] \mu \cosh (\mu L) \\
& \quad=-n \cosh (\mu L)\left( \pm B_{n-1, \mp}^{\mu,\left(\begin{array}{l}
4 \\
2
\end{array}\right)}-C_{n-1, \mp}^{\mu,\left(\begin{array}{l}
2 \\
4
\end{array}\right)}\right)=-n \cosh (\mu L)\left( \pm B_{n+1, \mp}^{\mu,\left(\begin{array}{l}
4 \\
2
\end{array}\right)}+C_{n+1, \mp}^{\left.\mu,\left(\begin{array}{l}
2 \\
4
\end{array}\right)\right)}\right) \text { for } n \geq 0, \mu \in \mathbb{R},
\end{aligned}
$$

where (3.36) and (3.37) are four (double-equality) relationships. Recall that the upper and the lower indices reflect the vanishing of $\widehat{\mathbf{x}}_{2}$ and $\widehat{\mathbf{x}}_{3}$ transversal components of the velocity field at $z=L$, respectively.

In order to proceed we have to simplify the velocity field (3.31) and the total pressure field (3.32) in terms of the expressions (3.36) and (3.37) for the constant coefficients. In view of that, we are primarily involved with the velocity (3.31), and we smartly manipulate certain group of terms (of the $\widehat{\mathbf{x}}_{2}$ and of the $\widehat{\mathbf{x}}_{3}$ components) with respect to (A.27), (A.28), as well as with a particular change of $n$, to obtain

$$
\begin{aligned}
& \sum_{n=0}^{\infty} \int \sum_{\mu}\left\{r J_{n}(\mu r)\left(B_{n, \mp}^{\mu,(2} 4\right) \cos \varphi \sin n \varphi \pm C_{n, \mp}^{\mu,\left(\frac{4}{2}\right)} \sin \varphi \cos n \varphi\right)
\end{aligned}
$$

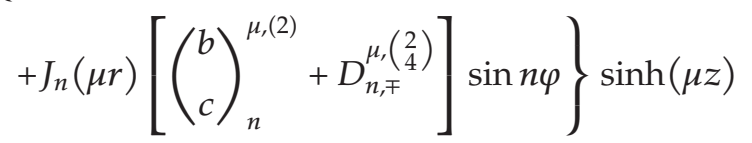

$$
\begin{aligned}
& =\sum_{n=0}^{\infty} \int \sum_{\mu} \frac{r}{2}\left\{\sin n \varphi \sinh (\mu z)\left[\left(B_{n-1, \mp}^{\mu,\left(\begin{array}{l}
2 \\
4
\end{array}\right)} \pm C_{n-1, \mp}^{\mu,\left(\begin{array}{l}
4 \\
2
\end{array}\right)}\right) J_{n-1}(\mu r)+\left(B_{n+1, \mp}^{\mu,\left(\begin{array}{l}
2 \\
4
\end{array}\right)} \mp C_{n+1, \mp}^{\mu,\left(\begin{array}{l}
4 \\
2
\end{array}\right)}\right) J_{n+1}(\mu r)\right]\right. \\
& \left.\left.+\sin n \varphi \sinh (\mu z)\left[\left(\begin{array}{l}
b \\
c
\end{array}\right)_{n}^{\mu,(2)}+D_{n, \mp}^{\mu,(2)} 4\right)\right] J_{n}(\mu r)\right\}
\end{aligned}
$$


Mathematical Problems in Engineering

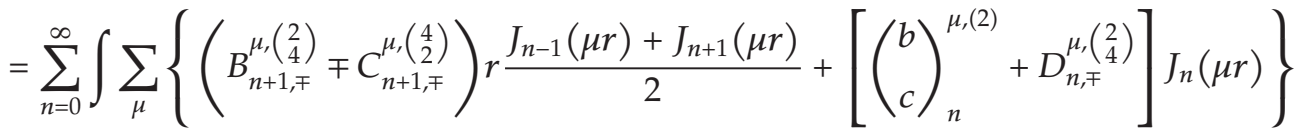

$$
\begin{aligned}
& \times \sin n \varphi \sinh (\mu z)
\end{aligned}
$$

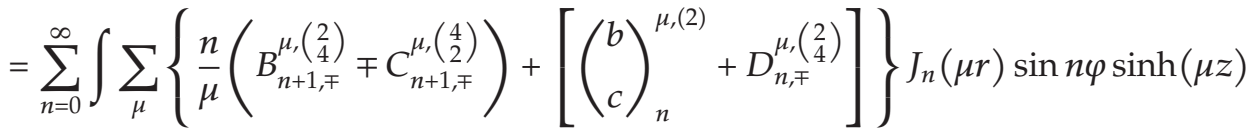

$$
\begin{aligned}
& =\sum_{n=0}^{\infty} \int \sum_{\mu}\left[-\frac{(\cosh (\mu L)+\mu L \sinh (\mu L))}{\mu \cosh (\mu L)} A_{n, \mp}^{\mu,\left(\begin{array}{l}
1 \\
3
\end{array}\right)}-\frac{(\sinh (\mu L)+\mu L \cosh (\mu L))}{\mu \cosh (\mu L)} A_{n, \mp}^{\left.\mu,\left(\begin{array}{l}
2 \\
4
\end{array}\right)\right]}\right. \\
& \times J_{n}(\mu r) \sin n \varphi \sinh (\mu z),
\end{aligned}
$$

where we have used the two equalities of relation (3.36) and the recurrence formula (A.23). Similarly, another group of terms of the velocity (3.31) shows that

$$
\begin{aligned}
& \sum_{n=0}^{\infty} \int \sum_{\mu}\left\{r J_{n}(\mu r)\left( \pm B_{n, \mp}^{\mu,\left(\begin{array}{l}
4 \\
2
\end{array}\right)} \cos \varphi \cos n \varphi+C_{n, \mp}^{\mu,\left(\begin{array}{l}
2 \\
4
\end{array}\right)} \sin \varphi \sin n \varphi\right)\right. \\
& \left.+J_{n}(\mu r)\left[\left(\begin{array}{l}
b \\
c
\end{array}\right)_{n}^{\mu,(4)} \pm D_{n, \mp}^{\mu,\left(\begin{array}{l}
4 \\
2
\end{array}\right)}\right] \cos n \varphi\right\} \sinh (\mu z) \\
& =\sum_{n=0}^{\infty} \int \sum_{\mu}\left[\mp \frac{(\cosh (\mu L)+\mu L \sinh (\mu L))}{\mu \cosh (\mu L)} A_{n, \mp}^{\mu,\left(\begin{array}{l}
3 \\
1
\end{array}\right)} \mp \frac{(\sinh (\mu L)+\mu L \cosh (\mu L))}{\mu \cosh (\mu L)} A_{n, \mp}^{\mu,\left(\begin{array}{l}
4 \\
2
\end{array}\right)}\right] \\
& \times J_{n}(\mu r) \cos n \varphi \sinh (\mu z) \text {, }
\end{aligned}
$$

where, here (we omitted common steps like those followed in (3.38)), we made use of (A.25), (A.26) and of relationship (3.37). Thus, we accomplished to simplify the $\widehat{\mathbf{x}}_{2}$-component and the $\widehat{\mathbf{x}}_{3}$-component of the velocity field (3.31). In addition, some further analysis on the second equalities of (3.36) and (3.37) gives

$$
\begin{gathered}
B_{n-1, \mp}^{\mu,\left(\begin{array}{l}
2 \\
4
\end{array}\right)} \pm C_{n-1, \mp}^{\mu,\left(\begin{array}{l}
4 \\
2
\end{array}\right)}=B_{n+1, \mp}^{\mu,\left(\begin{array}{l}
2 \\
4
\end{array}\right)} \mp C_{n+1, \mp}^{\mu,\left(\begin{array}{l}
4 \\
2
\end{array}\right)} \quad \text { with } B_{-1, \mp}^{\mu,\left(\begin{array}{l}
2 \\
4
\end{array}\right)}=C_{-1, \mp}^{\mu,\left(\begin{array}{l}
4 \\
2
\end{array}\right)} \equiv 0 \text { for } n \geq 0, \mu \in \mathbb{R}, \\
\pm B_{n-1, \mp}^{\mu,\left(\begin{array}{l}
4 \\
2
\end{array}\right)}-C_{n-1, \mp}^{\mu,\left(\begin{array}{l}
2 \\
4
\end{array}\right)}= \pm B_{n+1, \mp}^{\mu,\left(\begin{array}{l}
4 \\
2
\end{array}\right)}+C_{n+1, \mp}^{\mu,\left(\begin{array}{l}
2 \\
4
\end{array}\right)} \quad \text { with } B_{-1, \mp}^{\mu,\left(\begin{array}{l}
4 \\
2
\end{array}\right)}=C_{-1, \mp}^{\mu,\left(\begin{array}{l}
2 \\
4
\end{array}\right)} \equiv 0 \text { for } n \geq 0, \mu \in \mathbb{R},
\end{gathered}
$$

respectively, where in view of definitions (3.19)-(3.20) and after some trivial processing, expressions (3.40) and (3.41) take the form

$$
\begin{aligned}
& b_{n+1}^{\mu,(2)}-c_{n+1}^{\mu,(4)}=b_{n-1}^{\mu,(2)}+c_{n-1}^{\mu,(4)} \quad \text { with } b_{-1}^{\mu,(2)}=c_{-1}^{\mu,(4)} \equiv 0 \text { for } n \geq 0, \mu \in \mathbb{R}, \\
& b_{n+1}^{\mu,(4)}+c_{n+1}^{\mu,(2)}=b_{n-1}^{\mu,(4)}-c_{n-1}^{\mu,(2)} \quad \text { with } b_{-1}^{\mu,(4)}=c_{-1}^{\mu,(2)} \equiv 0 \text { for } n \geq 0, \mu \in \mathbb{R},
\end{aligned}
$$


respectively. The last equations (3.42), (3.43) provide us with the simplification of the third component of the velocity (3.31). Hence, proceeding to the $\widehat{\mathbf{x}}_{1}$-component of (3.31), for once more we utilize the relations (A.25)-(A.28), (A.23), and we apply a certain readjustment of the index $n$ so as to write

$$
\begin{aligned}
&-\frac{1}{2} \sum_{n=0} \int \sum_{\mu} \mu r J_{n}(\mu r) \cosh (\mu z) {\left[\left(b_{n}^{\mu,(2)} \cos \varphi+c_{n}^{\mu,(2)} \sin \varphi\right) \sin n \varphi\right.} \\
&\left.+\left(b_{n}^{\mu,(4)} \cos \varphi+c_{n}^{\mu,(4)} \sin \varphi\right) \cos n \varphi\right] \\
&=-\frac{1}{4} \sum_{n=0}^{\infty} \int \sum_{\mu} \mu r \cosh (\mu z)\left\{\sin n \varphi\left[\left(b_{n+1}^{\mu,(2)}-c_{n+1}^{\mu,(4)}\right) J_{n+1}(\mu r)+\left(b_{n-1}^{\mu,(2)}+c_{n-1}^{\mu,(4)}\right) J_{n-1}(\mu r)\right]\right. \\
&+\left.\cos n \varphi\left[\left(b_{n+1}^{\mu,(4)}+c_{n+1}^{\mu,(2)}\right) J_{n+1}(\mu r)+\left(b_{n-1}^{\mu,(4)}-c_{n-1}^{\mu,(2)}\right) J_{n-1}(\mu r)\right]\right\} \\
&=-\frac{1}{2} \sum_{n=0}^{\infty} \int \sum_{\mu} \mu r \cosh (\mu z)\left[\left(b_{n+1}^{\mu,(2)}-c_{n+1}^{\mu,(4)}\right) \sin n \varphi \frac{J_{n+1}(\mu r)+J_{n-1}(\mu r)}{2}\right.\left.+\left(b_{n+1}^{\mu,(4)}+c_{n+1}^{\mu,(2)}\right) \cos n \varphi \frac{J_{n+1}(\mu r)+J_{n-1}(\mu r)}{2}\right] \\
&=-\frac{1}{2} \sum_{n=0}^{\infty} \int \sum_{\mu} n J_{n}(\mu r) \cosh (\mu z)\left[\left(b_{n+1}^{\mu,(2)}-c_{n+1}^{\mu,(4)}\right) \sin n \varphi+\left(b_{n+1}^{\mu,(4)}+c_{n+1}^{\mu,(2)}\right) \cos n \varphi\right] .
\end{aligned}
$$

On the other hand, the total pressure (3.32) can also be simplified by using again definitions (3.19)-(3.20) and considering (3.42), (3.43) to evaluate

$$
\begin{aligned}
& -B_{n,-}^{\mu,(2)}-C_{n,+}^{\mu,(4)}=\frac{\mu}{2}\left[\left(b_{n+1}^{\mu,(2)}-c_{n+1}^{\mu,(4)}\right)-\left(b_{n-1}^{\mu,(2)}+c_{n-1}^{\mu,(4)}\right)\right]=0 \quad \text { for } n \geq 0, \mu \in \mathbb{R}, \\
& -B_{n,-}^{\mu,(4)}+C_{n,+}^{\mu,(2)}=\frac{\mu}{2}\left[\left(b_{n+1}^{\mu,(4)}+c_{n+1}^{\mu,(2)}\right)-\left(b_{n-1}^{\mu,(4)}-c_{n-1}^{\mu,(2)}\right)\right]=0 \quad \text { for } n \geq 0, \mu \in \mathbb{R} .
\end{aligned}
$$

Recapitulating the effect of the boundary condition (3.34), via (3.36) and (3.37), on the flow fields (3.31) and (3.32), we work as follows. In terms of (3.38), (3.39), (3.44), and (3.45), the only $b^{\prime}$ s, $c^{\prime}$ s, and $d^{\prime}$ s constant coefficients appear inside the $\widehat{\mathbf{x}}_{1}$-component of the velocity field (3.31), associated with each other as coefficients of the eigenfunctions $J_{n}(\mu r) \sin n \varphi \cosh (\mu z)$ and $J_{n}(\mu r) \cos n \varphi \cosh (\mu z)$ for every $n \geq 0$ and $\mu \in \mathbb{R}$. Since they appear nowhere else in the velocity or the total pressure, we can group them as

$$
\begin{aligned}
& e_{n}^{\mu_{,}(1)} \equiv-\mu d_{n}^{\mu,(2)}-n\left(b_{n+1}^{\mu,(2)}-c_{n+1}^{\mu,(4)}\right) \quad \text { for } n \geq 0, \mu \in \mathbb{R}, \\
& e_{n}^{\mu,(3)} \equiv-\mu d_{n}^{\mu,(4)}-n\left(b_{n+1}^{\mu,(4)}+c_{n+1}^{\mu,(2)}\right) \quad \text { for } n \geq 0, \mu \in \mathbb{R},
\end{aligned}
$$


without loss of generality. We insert the simplified relations (3.38), (3.39), and (3.44) into the velocity field (3.31), where with the aim of the new constant coefficients $e_{n}^{\mu,(1)}, e_{n}^{\mu,(3)}$ for $n \geq 0$ and $\mu \in \mathbb{R}$ provided by (3.46), (3.47), respectively, is furnished by

$$
\begin{array}{r}
\mathbf{v}=u(r) \widehat{\mathbf{z}}+\frac{\widehat{\mathbf{x}}_{1}}{2} \sum_{n=0}^{\infty} \int \sum_{\mu} J_{n}(\mu r)\left\{\operatorname { s i n } n \varphi \left[\left(a_{n}^{\mu,(1)}+e_{n}^{\mu,(1)}\right) \cosh (\mu z)+a_{n}^{\mu,(2)} \sinh (\mu z)\right.\right. \\
\left.-\mu z\left(a_{n}^{\mu,(1)} \sinh (\mu z)+a_{n}^{\mu,(2)} \cosh (\mu z)\right)\right] \\
+\cos n \varphi\left[\left(a_{n}^{\mu,(3)}+e_{n}^{\mu,(3)}\right) \cosh (\mu z)+a_{n}^{\mu,(4)} \sinh (\mu z)\right. \\
\left.\left.-\mu z\left(a_{n}^{\mu,(3)} \sinh (\mu z)+a_{n}^{\mu,(4)} \cosh (\mu z)\right)\right]\right\} \\
+\sum_{n=0}^{\infty} \int \sum_{\mu} \frac{J_{n}(\mu r)}{2 \mu}[(1+\mu L \tanh (\mu L)) \sinh (\mu z)-\mu z \cosh (\mu z)] \\
\times\left[-\left(A_{n,-}^{\mu,(1)} \sin n \varphi+A_{n,-}^{\mu,(3)} \cos n \varphi\right) \widehat{\mathbf{x}}_{2}+\left(A_{n,+}^{\mu,(1)} \cos n \varphi-A_{n,+}^{\mu,(3)} \sin n \varphi\right) \widehat{\mathbf{x}}_{3}\right] \\
+\sum_{n=0}^{\infty} \int \sum_{\mu} \frac{J_{n}(\mu r)}{2 \mu}[\tanh (\mu L)-\mu(z-L)] \sinh (\mu z) \\
\times\left[-\left(A_{n,-}^{\mu,(2)} \sin n \varphi+A_{n,-}^{\mu,(4)} \cos n \varphi\right) \widehat{\mathbf{x}}_{2}+\left(A_{n,+}^{\mu,(2)} \cos n \varphi-A_{n,+}^{\mu,(4)} \sin n \varphi\right) \widehat{\mathbf{x}}_{3}\right],
\end{array}
$$

while the total pressure field (3.32) with the aid of relations (3.45) becomes

$$
\begin{aligned}
P=P_{c}+p(z)-\eta X_{c} \sum_{n=0}^{\infty} \int \sum_{\mu} \mu J_{n}(\mu r)[ & \sin n \varphi\left(a_{n}^{\mu,(1)} \sinh (\mu z)+a_{n}^{\mu,(2)} \cosh (\mu z)\right) \\
& \left.+\cos n \varphi\left(a_{n}^{\mu,(3)} \sinh (\mu z)+a_{n}^{\mu,(4)} \cosh (\mu z)\right)\right],
\end{aligned}
$$

whereas the $A^{\prime}$ s constant coefficients are given through the $a^{\prime}$ s constant coefficients via (3.18), the factor $\chi_{c}$ satisfies (2.22), while $u(r)$ and $p(z)$ are given by relations (3.2). Hence, in order to complete our analytical method and obtain the final closed form of the flow fields (3.48) and (3.49), we have to calculate the rest of the constant coefficients, that is, the $a_{n}^{\mu,(1)}, a_{n}^{\mu,(2)}, a_{n}^{\mu,(3)}, a_{n}^{\mu,(4)}, e_{n}^{\mu,(1)}$, and $e_{n}^{\mu,(3)}$ for every $n \geq 0, \mu \in \mathbb{R}$ from the remaining boundary conditions.

Continuing, we handle the second part of the outlet boundary condition (2.30), and, as we mentioned earlier, we impose no height variations by setting $P \cong p$, in order to take

$$
P=\eta \frac{\partial(\widehat{\mathbf{z}} \cdot \mathbf{v})}{\partial z} \quad \text { for } z=L
$$


from which, with the contribution of the $z$-component of the velocity field (3.48) and the total pressure (3.49) (note that $\widehat{\mathbf{x}}_{1}=\widehat{\mathbf{z}}$, while expressions (3.2) denote $p(L)=0$ and $\partial u(r) / \partial z=0$ ), we obtain for $z=L$ the following condition:

$$
\begin{aligned}
P_{c}-\chi_{c} \sum_{n=0}^{\infty} \int \sum_{\mu} \mu J_{n}(\mu r)[ & \sin n \varphi\left(a_{n}^{\mu,(1)} \sinh (\mu L)+a_{n}^{\mu,(2)} \cosh (\mu L)\right) \\
+ & \left.\cos n \varphi\left(a_{n}^{\mu,(3)} \sinh (\mu L)+a_{n}^{\mu,(4)} \cosh (\mu L)\right)\right] \\
=\frac{1}{2} \sum_{n=0}^{\infty} \int \sum_{\mu} \mu J_{n}(\mu r)[ & \sin n \varphi\left(e_{n}^{\mu,(1)} \sinh (\mu L)-a_{n}^{\mu,(1)} \mu L \cosh (\mu L)-a_{n}^{\mu,(2)} \mu L \sinh (\mu L)\right) \\
& \left.+\cos n \varphi\left(e_{n}^{\mu,(3)} \sinh (\mu L)-a_{n}^{\mu,(3)} \mu L \cosh (\mu L)-a_{n}^{\mu,(4)} \mu L \sinh (\mu L)\right)\right] .
\end{aligned}
$$

Orthogonality arguments with respect to the eigenfunctions $J_{n}(\mu r) \sin n \varphi$ and $J_{n}(\mu r) \cos n \varphi$ for $n \geq 0$ and $\mu \in \mathbb{R}$ yield

$$
\begin{aligned}
& P_{c}=0,
\end{aligned}
$$

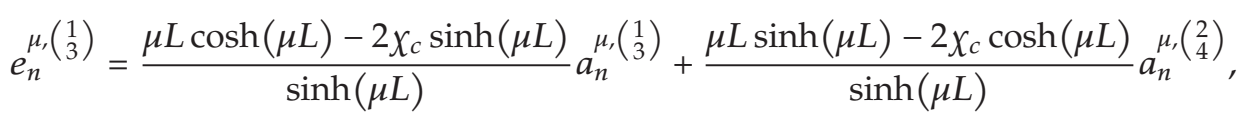

for $n \geq 0$ and $\mu \in \mathbb{R}$, where we have expressed the $e^{\prime}$ s constant coefficients via the $a^{\prime}$ s constant coefficients into the flow fields (3.48) and (3.49).

Consequently, we are left with four sets of constant coefficients; those are $a_{n}^{\mu,(1)}, a_{n}^{\mu,(2)}, a_{n}^{\mu,(3)}$, and $a_{n}^{\mu,(4)}$ for every $n \geq 0, \mu \in \mathbb{R}$, which must be determined from the continuity restraint (2.31) and the second part of the inlet boundary condition (2.29). The constant parameter $\mu \in \mathbb{R}$ will be evaluated from the nonslip boundary condition (2.28) in the end of this analysis. Afterwards we will verify that the limit (2.32) is valid. Although we deal with a well-posed boundary value problem, we observe that we have four conditions in order to calculate four constant coefficients and also parameter $\mu$. Hence, one constant coefficient will be undetermined. However, in view of a detailed analysis on the completeness of general solutions explained in [34] and also applied in [25], the differential representation used here (2.25) and (2.26) with (2.27), as well as the corresponding differential solutions [8-10], offers certain degrees of freedom to our solutions and permit the interrelation of the harmonic potentials $\Phi$ and $\Psi$ between each other in such a way so as to avoid any kind of indeterminacies to particular analytical solutions. This interrelation does not affect the generality of the analytical solutions (see, e.g., [25]), and it is applied to the constant coefficients. Therefore, we adopt the theory of [34], and we make a mathematical technique by choosing to express the $a^{\prime}$ s constant coefficients of kind $i=2,4$ in terms of those of kind $i=1,3$, via the relationships of proportionality

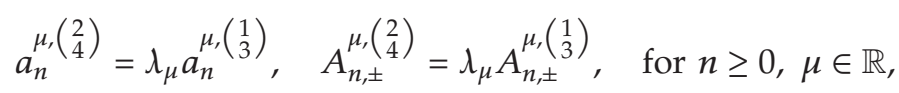


whereas $\lambda_{\mu} \in \mathbb{R}$ is the new parameter to be calculated for $\mu \in \mathbb{R}$, while the second equality of (3.54) is a consequence of definitions (3.18). Relationship (3.54) does not constrain the generality of our analytical method and at the same time will provide us with a closed unique solution. By substitution of relations (3.53) and (3.54) into the flow fields (3.48), (3.49), we can easily show that

$$
\begin{array}{r}
\mathbf{v}=u(r) \widehat{\mathbf{z}}+\frac{\widehat{\mathbf{x}}_{1}}{2} \sum_{n=0}^{\infty} \int \sum_{\mu} J_{n}(\mu r)\left(a_{n}^{\mu,(1)} \sin n \varphi+a_{n}^{\mu,(3)} \cos n \varphi\right) \\
\times\left\{-\mu z\left[\sinh (\mu z)+\lambda_{\mu} \cosh (\mu z)\right]+\lambda_{\mu} \sinh (\mu z)\right. \\
\left.+\left[\left(\mu L-2 x_{c} \lambda_{\mu}\right) \operatorname{coth}(\mu L)+\left(1-2 \chi_{c}\right)+\lambda_{\mu} \mu L\right] \cosh (\mu z)\right\} \\
+\sum_{n=0}^{\infty} \int \sum_{\mu} \frac{J_{n}(\mu r)}{2 \mu}\left\{\left[1+\left(\mu L+\lambda_{\mu}\right) \tanh (\mu L)-\lambda_{\mu} \mu(z-L)\right] \sinh (\mu z)-\mu z \cosh (\mu z)\right\} \\
\times\left[-\left(A_{n,-}^{\mu,(1)} \sin n \varphi+A_{n,-}^{\mu,(3)} \cos n \varphi\right) \widehat{\mathbf{x}}_{2}+\left(A_{n,+}^{\mu,(1)} \cos n \varphi-A_{n,+}^{\mu,(3)} \sin n \varphi\right) \widehat{\mathbf{x}}_{3}\right],
\end{array}
$$

for the velocity field and

$$
P=p(z)-\eta X_{c} \sum_{n=0}^{\infty} \int \sum_{\mu} \mu J_{n}(\mu r)\left(a_{n}^{\mu,(1)} \sin n \varphi+a_{n}^{\mu,(3)} \cos n \varphi\right)\left(\sinh (\mu z)+\lambda_{\mu} \cosh (\mu z)\right)
$$

for the total pressure field, respectively. Recall that the $A^{\prime}$ s constant coefficients are provided via the $a^{\prime}$ s constant coefficients from (3.18) and the factor $\chi_{c}$ is given through (2.22), while $u(r)$ and $p(z)$ assume the relations (3.2).

In order to impose the continuity restriction (2.31) at $z=L$, it would provide us with analytical convenience if we could write the velocity field (3.55) in pure cylindrical coordinates, meaning with the cylindrical unit vectors $\widehat{\mathbf{r}}, \widehat{\boldsymbol{\varphi}}, \widehat{\mathbf{z}}$. In view of that, it is obvious that $\widehat{\mathbf{x}}_{1}=\widehat{\mathbf{z}}$, while substituting the unit vectors in Cartesian coordinates $\widehat{\mathbf{x}}_{2}$ and $\widehat{\mathbf{x}}_{3}$ from (A.14), using (3.18), (A.22), (A.23), (A.25)-(A.28) and for one more time rearranging the index $n$ inside the series, we perform some analytical steps to express the Cartesian part of (3.55) in cylindrical coordinates as

$$
\begin{gathered}
\sum_{n=0}^{\infty} \int_{\mu} \frac{J_{n}(\mu r)}{2 \mu} Z_{\mu}(z)\left[-\left(A_{n,-}^{\mu,(1)} \sin n \varphi+A_{n,-}^{\mu,(3)} \cos n \varphi\right) \widehat{\mathbf{x}}_{2}+\left(A_{n,+}^{\mu,(1)} \cos n \varphi-A_{n,+}^{\mu,(3)} \sin n \varphi\right) \widehat{\mathbf{x}}_{3}\right] \\
=\frac{1}{2} \sum_{n=0}^{\infty} \int \sum_{\mu} Z_{\mu}(z)\left[\widehat{\mathbf{r}} \frac{d J_{n}(\mu r)}{d(\mu r)}\left(a_{n}^{\mu,(1)} \sin n \varphi+a_{n}^{\mu,(3)} \cos n \varphi\right)\right. \\
\left.+\widehat{\varphi} \frac{n J_{n}(\mu r)}{\mu r}\left(a_{n}^{\mu,(1)} \cos n \varphi-a_{n}^{\mu,(3)} \sin n \varphi\right)\right],
\end{gathered}
$$


where by definition

$$
Z_{\mu}(z)=\left[1+\left(\mu L+\lambda_{\mu}\right) \tanh (\mu L)-\lambda_{\mu} \mu(z-L)\right] \sinh (\mu z)-\mu z \cosh (\mu z),
$$

for $\lambda_{\mu} \in \mathbb{R}$ and $\mu \in \mathbb{R}$. By virtue of condition (2.31) in circular cylindrical coordinates (see the gradient from (A.15)) on the velocity (3.55), with the aim of (3.57) and definition (3.58), we conclude that

$$
\begin{aligned}
2 \chi_{c} \sum_{n=0}^{\infty} & \int \sum_{\mu} \mu J_{n}(\mu r)\left(a_{n}^{\mu,(1)} \sin n \varphi+a_{n}^{\mu,(3)} \cos n \varphi\right)\left[\sinh (\mu L)+\lambda_{\mu} \cosh (\mu L)\right] \\
& =-\sum_{n=0}^{\infty} \int \sum_{\mu} \mu J_{n}(\mu r)\left(a_{n}^{\mu,(1)} \sin n \varphi+a_{n}^{\mu,(3)} \cos n \varphi\right) \frac{\sinh (\mu L) \cosh (\mu L)-\mu L+\lambda_{\mu} \sinh ^{2}(\mu L)}{\cosh (\mu L)},
\end{aligned}
$$

where we have used the Bessel ordinary differential equation (A.18) to evaluate

$$
\frac{d^{2} J_{n}(\mu r)}{d(\mu r)^{2}}+\frac{1}{(\mu r)} \frac{d J_{n}(\mu r)}{d(\mu r)}-\frac{n^{2}}{(\mu r)^{2}} J_{n}(\mu r)=-J_{n}(\mu r) \quad \text { for } \mu \in \mathbb{R}
$$

Simple orthogonality arguments of the products $\mu J_{n}(\mu r)\left(a_{n}^{\mu,(1)} \sin n \varphi+a_{n}^{\mu,(3)} \cos n \varphi\right)$ for every $n \geq 0$ and $\mu \in \mathbb{R}$ on (3.59) provides us with the constant parameter $\lambda_{\mu}$ as

$$
\lambda_{\mu}=\frac{\mu L-2^{-1}\left(2 \chi_{c}+1\right) \sinh (2 \mu L)}{\left(2 \chi_{c}+1\right) \cosh ^{2}(\mu L)-1} \quad \text { with } \sinh (2 \mu L)=2 \sinh (\mu L) \cosh (\mu L) \text { for } \mu \in \mathbb{R} \text {. }
$$

Actually, the flow fields retain only the parameter $\mu$, and their equivalent form with respect to $(3.61)$ is

$$
\begin{array}{r}
\mathbf{v}=u(r) \widehat{\mathbf{z}}+\widehat{\mathbf{x}}_{1} \sum_{n=0}^{\infty} \int \sum_{\mu} J_{n}(\mu r) g_{\mu}(\mu z)\left(a_{n}^{\mu,(1)} \sin n \varphi+a_{n}^{\mu,(3)} \cos n \varphi\right) \\
+\sum_{n=0}^{\infty} \int \sum_{\mu} \frac{J_{n}(\mu r)}{\mu} g_{\mu}^{\prime}(\mu z)\left[-\left(A_{n,-}^{\mu,(1)} \sin n \varphi+A_{n,-}^{\mu,(3)} \cos n \varphi\right) \widehat{\mathbf{x}}_{2}\right. \\
\left.+\left(A_{n,+}^{\mu,(1)} \cos n \varphi-A_{n,+}^{\mu,(3)} \sin n \varphi\right) \widehat{\mathbf{x}}_{3}\right]
\end{array}
$$

from velocity field (3.55) and

$$
P=p(z)+\sum_{n=0}^{\infty} \int \sum_{\mu} J_{n}(\mu r) h_{\mu}(\mu z)\left(a_{n}^{\mu,(1)} \sin n \varphi+a_{n}^{\mu,(3)} \cos n \varphi\right)
$$


from total pressure field (3.56), respectively, where the prime appearing at $\mathrm{g}_{\mu}^{\prime}(\mu \mathrm{z})$ denotes derivation with respect to the variable $\mu z \in \mathbb{R}$. The new functions of expansions (3.62) and (3.63) are defined as

$$
\begin{aligned}
g_{\mu}(\mu z)=\frac{1}{2}[ & \frac{\mu L-2^{-1}\left(2 \chi_{c}+1\right) \sinh (2 \mu L)}{\left(2 \chi_{c}+1\right) \cosh ^{2}(\mu L)-1} \sinh (\mu z) \\
& \left.\quad+\left(1+\frac{2 \chi_{c}+(\mu L)^{2}}{\left(2 \chi_{c}+1\right) \cosh ^{2}(\mu L)-1}\right) \cosh (\mu z)+\frac{z}{\eta \chi_{c}} h_{\mu}(\mu z)\right] \quad \text { for } \mu \in \mathbb{R},
\end{aligned}
$$

where

$$
h_{\mu}(\mu z)=-\eta \chi_{c} \mu\left[\sinh (\mu z)+\frac{\mu L-2^{-1}\left(2 \chi_{c}+1\right) \sinh (2 \mu L)}{\left(2 \chi_{c}+1\right) \cosh ^{2}(\mu L)-1} \cosh (\mu z)\right] \quad \text { for } \mu \in \mathbb{R} \text {. }
$$

Here, we must recall that the $A^{\prime}$ s constant coefficients are given through the $a^{\prime}$ s constant coefficients via (3.18) and the factor $\chi_{c}$ is provided by (2.22), while $u(r)$ and $p(z)$ assume the well-known expressions (3.2).

One can easily manipulate relationships (3.64) and (3.65) to prove that, for $z=L$,

$$
\lim _{L \rightarrow+\infty} h_{\mu}(\mu L)=\lim _{L \rightarrow+\infty} h_{\mu}^{\prime}(\mu L)=0, \quad \lim _{L \rightarrow+\infty} g_{\mu}(\mu L)=\lim _{L \rightarrow+\infty} g_{\mu}^{\prime}(\mu L)=0, \quad \text { for } \mu \in \mathbb{R},
$$

and provided that the remaining unknown constant coefficients $a_{n}^{\mu,(1)}$ and $a_{n}^{\mu,(3)}$ for $n \geq 0$, as well as the unknown parameter $\mu \in \mathbb{R}$, are bounded, the velocity (3.62) and total pressure (3.63) asymptotically lead to

$$
\lim _{L \rightarrow+\infty} \mathbf{v}=u(r) \widehat{\mathbf{z}}, \quad \lim _{L \rightarrow+\infty} \frac{\partial P}{\partial z}=\frac{d p(L)}{d z} \text { for } z=L \longrightarrow+\infty
$$

Hence, we obtain the predictable parabolic profile of the Poiseuille flow (3.2), and, consequently, the asymptotic limit (2.32) holds true.

Our next step involves the determination of the constant parameter $\mu$, which will be calculated by the nonslip condition (2.28), where the velocity (3.62) vanishes $(\mathbf{v}=0)$ at $r=\alpha$, that is,

$$
\begin{aligned}
\widehat{\mathbf{x}}_{1} \sum_{n=0}^{\infty} \int \sum_{\mu} J_{n}(\mu \alpha) g_{\mu}(\mu z)\left(a_{n}^{\mu,(1)} \sin n \varphi+a_{n}^{\mu,(3)} \cos n \varphi\right) \\
+\sum_{n=0}^{\infty} \int \sum_{\mu} \frac{J_{n}(\mu \alpha)}{\mu} g_{\mu}^{\prime}(\mu z)\left[-\left(A_{n,-}^{\mu,(1)} \sin n \varphi+A_{n,-}^{\mu,(3)} \cos n \varphi\right) \widehat{\mathbf{x}}_{2}\right. \\
\left.+\left(A_{n,+}^{\mu,(1)} \cos n \varphi-A_{n,+}^{\mu,(3)} \sin n \varphi\right) \widehat{\mathbf{x}}_{3}\right]=\mathbf{0},
\end{aligned}
$$


which is a three-component condition, and each component must be set to nil at $r=\alpha$. In the aim of satisfying all the three conditions from (3.68) at the same time, we easily observe that it is required the nihilism of the Bessel functions on the surface of the cylinder, that is,

$$
J_{n}(\mu \alpha)=0 \text { for } n \geq 0, \mu \in \mathbb{R},
$$

a condition that provides us with the value of the parameter $\mu$ as discrete eigenvalues via

$$
\mu \equiv \mu_{n}^{m}=\frac{\rho_{n}^{m}}{\alpha} \quad \text { for } n \geq 0, m \geq 1,
$$

where $\rho_{n}^{m}$ is the $m$-root $(m \geq 1)$ of order $n \geq 0$ of the Bessel functions $\left(J_{n}\left(\rho_{n}^{m}\right)=0\right)$. Consequently, formula (3.70) determines the parameter $\mu \in \mathbb{R}$, which is rather an expected result for such kind of problems. Since the constant parameter $\mu$ takes discrete values, it is obvious that the defined symbol " $\int \Sigma_{\mu} \ldots$ " used inside the fields (3.62), (3.63) must be substituted by the standard series symbol " $\sum_{m=1}^{\infty} \ldots$ ". Thus, the orthogonality relation for the Bessel functions (A.32) can be applied in our forthcoming calculations at which we will retain the $\mu_{n}^{m}$-symbolism given now by (3.70), where $\mu_{n}^{m}$ is the $m$-parameter $(m \geq 1)$ of order $n \geq 0$. Introducing the new constant coefficients that have to be calculated as

$$
a_{n}^{m} \equiv a_{n}^{\mu_{,}(1)}, \quad b_{n}^{m} \equiv a_{n}^{\mu_{,}(3)} \quad \text { for } n \geq 0, m \geq 1, \mu \equiv \mu_{n}^{m} \in \mathbb{R},
$$

and instead of (3.18) their equivalent forms

$$
\begin{aligned}
& A_{n, \pm}^{m} \equiv \frac{1}{\mu} A_{n, \pm}^{\mu,(1)}=\frac{1}{2}\left(a_{n-1}^{\mu,(1)} \pm a_{n+1}^{\mu,(1)}\right) \equiv \frac{1}{2}\left(a_{n-1}^{m} \pm a_{n+1}^{m}\right) \quad \text { with } a_{-1}^{\mu,(1)} \equiv a_{-1}^{m} \equiv 0 \\
& B_{n, \pm}^{m} \equiv \frac{1}{\mu} A_{n, \pm}^{\mu,(3)}=\frac{1}{2}\left(a_{n-1}^{\mu,(3)} \pm a_{n+1}^{\mu,(3)}\right) \equiv \frac{1}{2}\left(b_{n-1}^{m} \pm b_{n+1}^{m}\right) \quad \text { with } a_{-1}^{\mu,(3)} \equiv b_{-1}^{m} \equiv 0
\end{aligned}
$$

for every $n \geq 0, m \geq 1$, and $\mu \equiv \mu_{n}^{m} \in \mathbb{R}$, the velocity field (3.62) is given via the eigenvaluetype expansion

$$
\begin{aligned}
\mathbf{v}= & u(r) \widehat{\mathbf{z}}+\widehat{\mathbf{x}}_{1} \sum_{n=0}^{\infty} \sum_{m=1}^{\infty} J_{n}\left(\mu_{n}^{m} r\right) g_{n}^{m}\left(\mu_{n}^{m} z\right)\left(a_{n}^{m} \sin n \varphi+b_{n}^{m} \cos n \varphi\right) \\
& +\sum_{n=0}^{\infty} \sum_{m=1}^{\infty} J_{n}\left(\mu_{n}^{m} r\right) \frac{d g_{n}^{m}\left(\mu_{n}^{m} z\right)}{d\left(\mu_{n}^{m} z\right)}\left[-\left(A_{n,-}^{m} \sin n \varphi+B_{n,-}^{m} \cos n \varphi\right) \widehat{\mathbf{x}}_{2}+\left(A_{n,+}^{m} \cos n \varphi-B_{n,+}^{m} \sin n \varphi\right) \widehat{\mathbf{x}}_{3}\right],
\end{aligned}
$$

while the total pressure field (3.63), under the aforementioned readjustment, gives rise to

$$
P=p(z)+\sum_{n=0}^{\infty} \sum_{m=1}^{\infty} J_{n}\left(\mu_{n}^{m} r\right) h_{n}^{m}\left(\mu_{n}^{m} z\right)\left(a_{n}^{m} \sin n \varphi+b_{n}^{m} \cos n \varphi\right)
$$


The functions (3.64) and (3.65), which are involved into the flow fields (3.73) and (3.74), are now provided by the following formulae:

$$
\begin{aligned}
g_{n}^{m}\left(\mu_{n}^{m} z\right)=\frac{1}{2}[ & \frac{\mu_{n}^{m} L-2^{-1}\left(2 \chi_{c}+1\right) \sinh \left(2 \mu_{n}^{m} L\right)}{\left(2 \chi_{c}+1\right) \cosh ^{2}\left(\mu_{n}^{m} L\right)-1} \sinh \left(\mu_{n}^{m} z\right)+\left(1+\frac{2 \chi_{c}+\left(\mu_{n}^{m} L\right)^{2}}{\left(2 \chi_{c}+1\right) \cosh ^{2}\left(\mu_{n}^{m} L\right)-1}\right) \\
& \left.\times \cosh \left(\mu_{n}^{m} z\right)+\frac{z}{\eta \chi_{c}} h_{n}^{m}\left(\mu_{n}^{m} z\right)\right] \text { for } n \geq 0, m \geq 1,
\end{aligned}
$$

where

$$
\begin{array}{r}
h_{n}^{m}\left(\mu_{n}^{m} z\right)=-\eta \chi_{c} \mu_{n}^{m}\left[\sinh \left(\mu_{n}^{m} z\right)+\frac{\mu_{n}^{m} L-2^{-1}\left(2 \chi_{c}+1\right) \sinh \left(2 \mu_{n}^{m} L\right)}{\left(2 \chi_{c}+1\right) \cosh ^{2}\left(\mu_{n}^{m} L\right)-1} \cosh \left(\mu_{n}^{m} z\right)\right] \\
\text { for } n \geq 0, m \geq 1 .
\end{array}
$$

Here, $\mu_{n}^{m}, \chi_{c}$ and $u(r), p(z)$ satisfy relations (3.70), (2.22), and (3.2), respectively, while the remaining constant coefficients $a_{n}^{m}$ and $b_{n}^{m}$ for $n \geq 0$ and $m \geq 1$ will be determined from the second part of boundary condition (2.29), which is the last one to deal with.

Straightforward application of the imposed entrance velocity $\widehat{\mathbf{z}} \cdot \mathbf{v}=v(r, \varphi)$ for $z=0$ at the velocity (3.73) (note that $\widehat{\mathbf{x}}_{1}=\widehat{\mathbf{z}}$ ) and use of (3.70) result in

$$
u(r)+\sum_{n=0}^{\infty} \sum_{m=1}^{\infty} J_{n}\left(\rho_{n}^{m} \frac{r}{\alpha}\right) g_{n}^{m}(0)\left(a_{n}^{m} \sin n \varphi+b_{n}^{m} \cos n \varphi\right)=v(r, \varphi)
$$

By virtue of the orthogonality relation (A.32) and the well-known orthogonality of the trigonometric functions, relation (3.77) reveals that

$$
\left\{\begin{array}{l}
a_{n}^{m} \\
b_{n}^{m}
\end{array}\right\}=\varepsilon_{n}\left[\frac{\pi \alpha^{2}}{2} g_{n}^{m}(0) J_{n+1}^{2}\left(\rho_{n}^{m}\right)\right]^{-1} \int_{0}^{2 \pi} \int_{0}^{\alpha}[v(r, \varphi)-u(r)] J_{n}\left(\rho_{n}^{m} \frac{r}{\alpha}\right)\left\{\begin{array}{l}
\sin n \varphi \\
\cos n \varphi
\end{array}\right\} r d r d \varphi,
$$

for $n \geq 0$ and $m \geq 1$, where $\varepsilon_{n}=2$ for $n \geq 1, \varepsilon_{0}=1$ and $u(r)$ is given in (3.2). It is trivial to check the nice behavior of the constant coefficients (3.78) as $L \rightarrow+\infty$. Relations (3.78) coincide with (3.72) and provide us with the final form of the flow fields (3.73) and (3.74).

Two basic special cases are the following. If the imposed velocity is only a function of the variable $r$, that is, $v(r)$, then from relations (3.78) the only constant coefficients that survive are the $b_{0}^{m}$ for $m \geq 1$ and the magnetic flow becomes axisymmetric. Moreover, in this case, if $v(r)=u(r)$, then (3.78) shows that $a_{n}^{m}=b_{n}^{m}=0$ for $n \geq 0$ and $m \geq 1$; thus, the magnetic flow (3.73) becomes the magnetic Poiseuille flow $\mathbf{v}=u(r) \widehat{\mathbf{z}}$.

In the sense of making this work more complete, we follow some easy analytical steps, identical to those followed for the production of relationship (3.57), with extensive use of formulae (A.25)-(A.28) and with a proper rearranging of the index $n \geq 0$, in order to write the velocity (3.73) in pure circular cylindrical coordinates with unit vectors $\widehat{\mathbf{r}}, \widehat{\boldsymbol{\varphi}}$, and $\widehat{\mathbf{z}}$. In 
view of this aim, we substitute the Cartesian unit vectors $\widehat{\mathbf{x}}_{i}, i=1,2,3$, from (A.14) into (3.73), and we utilize expressions (3.72) in terms of the functions

$$
\begin{gathered}
j_{n, \pm}^{m}\left(\mu_{n}^{m} r, \mu_{n}^{m} z\right)=\frac{1}{2}\left[J_{n-1}\left(\mu_{n-1}^{m} r\right) \frac{d g_{n-1}^{m}\left(\mu_{n-1}^{m} z\right)}{d\left(\mu_{n-1}^{m} z\right)} \pm J_{n+1}\left(\mu_{n+1}^{m} r\right) \frac{d g_{n+1}^{m}\left(\mu_{n+1}^{m} z\right)}{d\left(\mu_{n+1}^{m} z\right)}\right] \text { for } n \geq 0, \\
f_{n}^{m}(n \varphi)=a_{n}^{m} \sin n \varphi+b_{n}^{m} \cos n \varphi \quad \text { for } n \geq 0, m \geq 1
\end{gathered}
$$

where $\mu_{n}^{m}=\rho_{n}^{m} / \alpha\left(\rho_{n}^{m}\right.$ being the $m$-root $(m \geq 1)$ of order $n \geq 0$ of the Bessel functions). Thus, we obtain the equivalent form of the velocity field (3.73)

$$
\begin{aligned}
\mathbf{v}(r, \varphi, z)= & {\left[u(r)+\sum_{n=0}^{\infty} \sum_{m=1}^{\infty} J_{n}\left(\rho_{n}^{m} \frac{r}{\alpha}\right) g_{n}^{m}\left(\rho_{n}^{m} \frac{z}{\alpha}\right) f_{n}^{m}(n \varphi)\right] \widehat{\mathbf{z}} } \\
& +\sum_{n=0}^{\infty} \sum_{m=1}^{\infty}\left[j_{n,-}^{m}\left(\rho_{n}^{m} \frac{r}{\alpha}, \rho_{n}^{m} \frac{z}{\alpha}\right) f_{n}^{m}(n \varphi) \widehat{\mathbf{r}}+j_{n,+}^{m}\left(\rho_{n}^{m} \frac{r}{\alpha}, \rho_{n}^{m} \frac{z}{\alpha}\right) \frac{d f_{n}^{m}(n \varphi)}{d(n \varphi)} \widehat{\boldsymbol{\varphi}}\right],
\end{aligned}
$$

and of the total pressure field (3.74)

$$
P(r, \varphi, z)=p(z)+\sum_{n=0}^{\infty} \sum_{m=1}^{\infty} J_{n}\left(\rho_{n}^{m} \frac{r}{\alpha}\right) h_{n}^{m}\left(\rho_{n}^{m} \frac{z}{\alpha}\right) f_{n}^{m}(n \varphi)
$$

Recapitulating, we completed our analytical method with the application of all the required boundary conditions of the particular physical problem that we had to solve. Hence, given a known velocity field $v(r, \varphi)$ at the entrance of the circular pipe, the velocity, and the total pressure of a magnetic fluid (perturbed by the three-dimensional constant magnetic field (2.20)) within the prescribed dimensions

$$
V\left(\mathbb{R}^{3}\right)=\{(r, \varphi, z): 0 \leq r \leq \alpha, 0 \leq \varphi<2 \pi, 0 \leq z \leq L\},
$$

assume the expansions (3.81) and (3.82), respectively, where $a_{n}^{m}, b_{n}^{m}$ for $n \geq 0$ and $m \geq 1$ have been calculated through relations (3.78). The functions $h_{n}^{m}\left(\mu_{n}^{m} z\right), g_{n}^{m}\left(\mu_{n}^{m} z\right), j_{n, \pm}^{m}\left(\mu_{n}^{m} r, \mu_{n}^{m} z\right)$, and $f_{n}^{m}(n \varphi)$ for $n \geq 0$ and $m \geq 1$ are given by (3.75), (3.76), (3.79), and (3.80), respectively, while $\mu_{n}^{m}=\rho_{n}^{m} / \alpha, X_{c}$ and $u(r), p(z)$ satisfy relationships (3.70), (2.22), and (3.2), respectively. Since $\rho_{n}^{m}$ is the $m$-root $(m \geq 1)$ of order $n \geq 0$ of the Bessel functions, that is, $J_{n}\left(\rho_{n}^{m}\right)=0$, then obviously $j_{n, \pm}^{m}\left(\rho_{n}^{m}, \mu_{n}^{m} z\right)=0$. Finally, any information needed beyond this section, which concerns the Bessel functions $J_{n}\left(\mu_{n}^{m} r\right)$ or the trigonometric and hyperbolic functions, is collected into the appendix.

\section{Numerical Results and Discussion}

The problem under consideration is the creeping flow inside a straight circular tube under the influence of a constant vector uniform magnetic field arbitrarily orientated. The radius of 
the pipe is $\alpha$, and its length is $L$. The mean axial velocity of the flow at the inlet of the pipe is given by the relation

$$
U=\frac{\iint_{\Lambda} v(r, \varphi) r d r d \varphi}{\iint_{\Lambda} r d r d \varphi}
$$

where $\Lambda$ is the cross section at the inlet. Consequently, the mass flow rate at the inlet of the duct is

$$
\dot{m}=\rho U \Lambda \text {. }
$$

If we consider the limiting case of a semi-infinite duct with $L \rightarrow+\infty$ and apply mass conservation at the inlet and the outlet of the duct, then it is obtained that the mass rate at which the fluid enters the cylinder must be equal to the mass rate at the exit. Taking into account the analysis presented in (2.32), we obtain a relation that expresses the axial pressure gradient $-A$ in terms of the mean axial velocity at the inlet $U$, through the fully developed velocity profile $u(r)$ of (3.2) as

$$
\dot{m}=\rho U \Lambda=\rho \frac{\iint_{\Lambda} u(r) r d r d \varphi}{\iint_{\Lambda} r d r d \varphi} \Lambda=\rho \frac{A \alpha^{2}}{8 \eta \chi_{c}} \Lambda
$$

which yields

$$
A=\frac{8 U \eta X_{c}}{\alpha^{2}}
$$

In order for our results to have general applicability, we introduce the nondimensional variables

$$
\begin{gathered}
\overline{\mathbf{v}}=\frac{\mathbf{v}}{U^{\prime}} \quad \bar{v}(r, \varphi)=\frac{v(r, \varphi)}{U}, \quad \bar{u}(r)=\frac{u(r)}{U}, \quad \bar{r}=\frac{r}{\alpha}, \\
\bar{z}=\frac{z}{\alpha}, \quad \bar{L}=\frac{L}{\alpha}, \quad \bar{P}=\frac{P}{\eta U / \alpha}, \quad \bar{A}=\frac{A}{\eta U / \alpha^{2}} .
\end{gathered}
$$

Thus, we can apply solution (3.81) and (3.82) to the problem of developing flow of a micropolar fluid in the entrance region of a circular duct to obtain the corresponding flow field. In all the cases that we examined in the summations of (3.81) and (3.82), we used the first 18 terms.

In the first case the inlet velocity profile was chosen to be

$$
v(r, \varphi)=1-r^{5},
$$

which is very close to a uniform inlet velocity. Figure 1 shows contour plots of the dimensionless axial velocity component $\overline{\mathbf{u}}_{z} \equiv \widehat{\mathbf{z}} \cdot \overline{\mathbf{v}}$, the dimensionless radial velocity component 


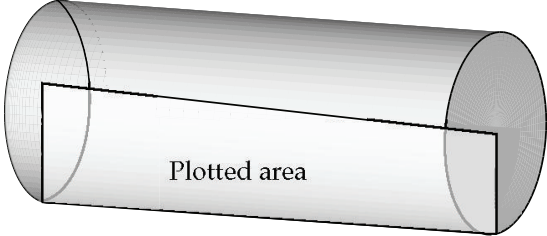

(a)

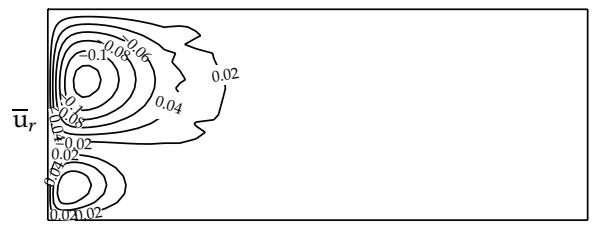

(c)

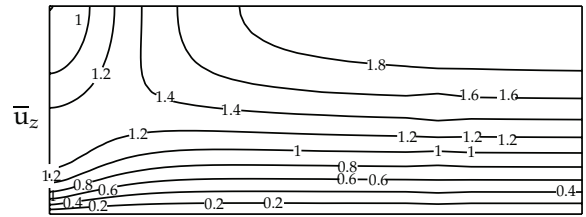

(b)

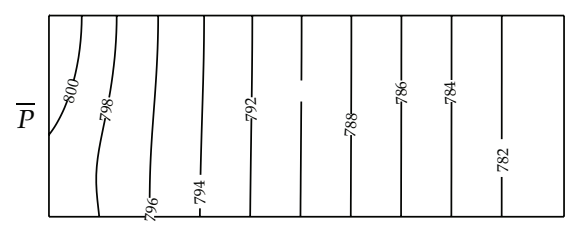

(d)

Figure 1: Contour plots of the dimensionless axial velocity component $\overline{\mathrm{u}}_{z}$, the dimensionless radial velocity component $\overline{\mathrm{u}}_{r}$, and the dimensionless total pressure $\bar{P}$ over a longitudinal-radial section of the duct.

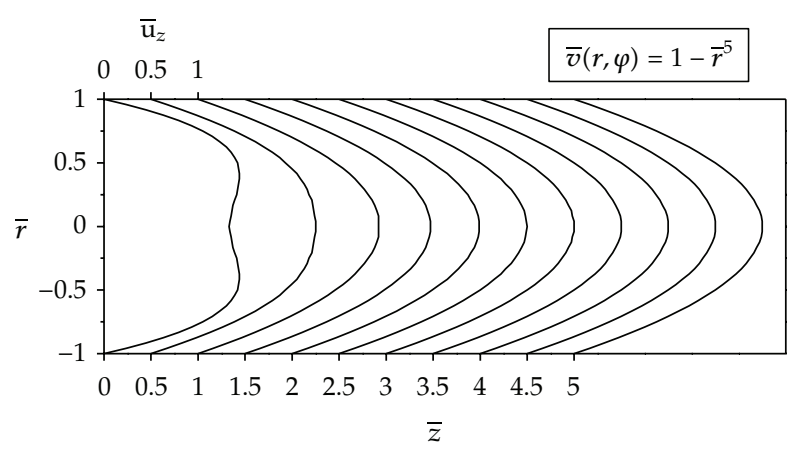

Figure 2: Diagrams of the dimensionless axial velocity distribution $\overline{\mathrm{u}}_{z}$ along the radial direction for different axial lengths $\bar{z}$.

$\overline{\mathbf{u}}_{r} \equiv \widehat{\mathbf{r}} \cdot \overline{\mathbf{v}}$, and the dimensionless total pressure $\bar{P}$. The plots are taken in a longitudinal-radial section of the duct, as shown in Figure 1.

It is observed that, as the flow moves downstream, it becomes fully developed. The radial velocity vanishes, the pressure drop becomes constant and uniform over the crosssection of the duct, and the axial velocity obtains a parabolic form. This last observation is clearer in the plots of Figure 2 that depict the development of the axial velocity in the longitudinal direction.

This figure shows the dimensionless axial velocity distribution $\overline{\mathrm{u}}_{z}$ along the radial direction for different axial lengths. It is seen that the axial velocity has initially the inlet profile (4.6), which gradually transforms to the parabolic profile. It must be noted, however, that the inlet profile is slightly distorted in the area close to the center at $r=0$, where we see a small reduction of the axial velocity although the inlet profile (4.6) is nondecreasing. This is attributed to the function of the inlet profile, which changes very rapidly with $r$. 


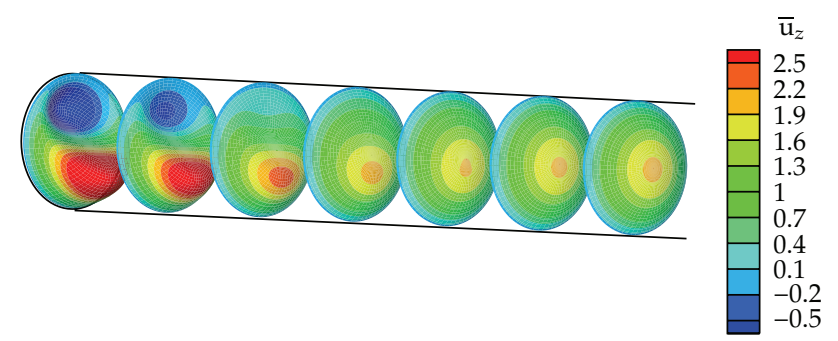

(a)

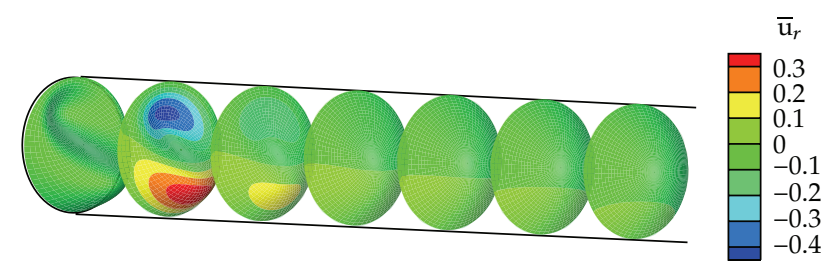

(b)

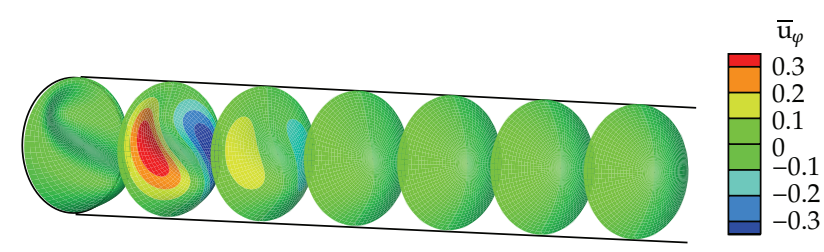

(c)

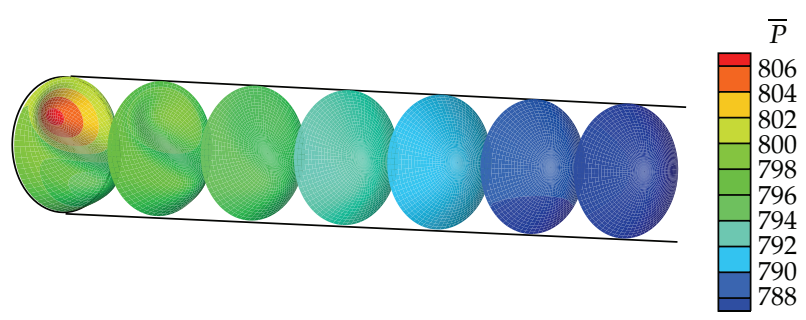

(d)

Figure 3: Contour plots of the dimensionless axial velocity component $\overline{\mathrm{u}}_{z}$, the dimensionless radial velocity component $\overline{\mathrm{u}}_{r}$, the dimensionless angular velocity component $\overline{\mathrm{u}}_{\varphi}$, and the dimensionless total pressure $\bar{P}$ at several downstream locations.

Since the solution that we present is able to predict 3D flow without any symmetries, we chose to examine a flow with a nonsymmetrical profile. Thus, we imposed an inlet velocity profile given by the function

$$
v(r, \varphi)=1-r^{5}+\sin (\pi r) \sin \varphi
$$

Figure 3 shows contours of the dimensionless axial velocity component $\bar{u}_{z}$, the dimensionless radial velocity component $\overline{\mathrm{u}}_{r}$, the dimensionless angular velocity component $\overline{\mathrm{u}}_{\varphi} \equiv \hat{\boldsymbol{\varphi}} \cdot \overline{\mathrm{v}}$, and the dimensionless total pressure $\bar{P}$, at several downstream locations. 


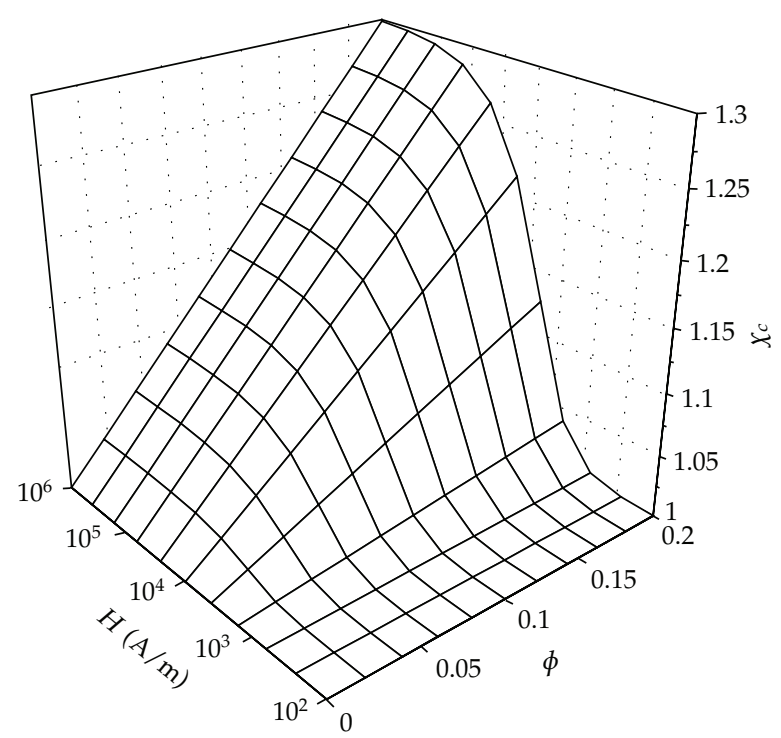

Figure 4: Variation of parameter $\chi_{c}$ with the magnetic field $H$ and the volumetric concentration of magnetic particles $\phi$, where the plot corresponds to fully developed flow of a micropolar fluid.

A general remark is that, as the flow enters the pipe, there is intense movement of the fluid in the cross-sectional plane, which gradually leads to a fully developed velocity. The pressure distribution, which is initially highly nonuniform, changes as the flow moves downstream, and it eventually reaches a uniform profile.

Another interesting characteristic of micropolar flow is the increase of the viscosity, and consequently of the friction losses, due to the magnetic field. In order to investigate these effects, we define the Reynolds number Re and the Fanning friction factor $f$ as in [35]:

$$
\operatorname{Re}=\frac{\rho U D_{h}}{\eta}, \quad f=\frac{\tau_{w}}{\rho U^{2} / 2}
$$

where $D_{h}=2 \alpha$ is the hydraulic diameter and $\tau_{w}$ is wall shear stress. For fully developed flow in a circular duct, the wall shear stress is related to the axial pressure drop via the relation $2 \tau_{w}=-\alpha d P_{\mathrm{P}} / d z=\alpha A$. Applying this relation to (4.8) and using (4.4), we obtain the relation for the friction factor $f$ product with the Reynolds number

$$
f \operatorname{Re}=16 \chi_{c},
$$

which is valid for fully developed magnetic flow in a circular duct. If there are no magnetic particles or if there is no magnetic field, then $\chi_{c}=1$ and (4.9) gives the well-known relation $f \operatorname{Re}=16$. Thus, in order to assess the additional friction losses due to the magnetic field, we examine the parameter $x_{c}$.

Figure 4 depicts the dependence of $X_{c}$ on the magnetic field $H$ and on the volumetric concentration of magnetic particles $\phi$, which is defined in relation (2.4).

The plots correspond to constant temperature $T=300 \mathrm{~K}$, magnetic dipole strength $m=$ $2 \times 10^{-18} \mathrm{~A} \cdot \mathrm{m}^{2}$, viscosity $\eta=10^{-3} \mathrm{~kg} / \mathrm{ms}$, and small magnetic particles of radius $r_{p}<10^{-7} \mathrm{~m}$ 
so that the assumptions that were made for the derivation of the Stokes equations are valid. It is noted that the parameter $\chi_{c}$ does not depend on the particle radius $r_{p}$. This can be seen from the relation (2.22) after the substitution of $\tau_{S}, \tau_{B}, I$ and of $M_{0, c}$ from (2.21). From the plot of Figure 4 it is observed that, independently of the concentration $\phi$, the parameter $\chi_{c}$ undergoes a steep increase in the interval $10^{3}<H<10^{5}(\mathrm{~A} / \mathrm{m})$, while it remains steady for smaller or higher values of the magnetic field. Moreover, it is observed that the parameter $X_{c}$ increases in a linear manner as the concentration $\phi$ increases.

\section{Conclusions}

Many applications of practical interest in modern engineering technology deal with the micropolar magnetohydrodynamic flow of magnetic fluids in the presence of magnetic fields. In this paper we examined how a 3D constant uniform magnetic field perturbs a threedimensional steady creeping motion (Stokes flow) of a viscous incompressible micropolar fluid of approximately zero conductivity in a circular cylinder of a finite length. The ferrofluid was considered to be a colloidal suspension of nonconductive ferromagnetic material of very small spherical particles, which follow the Brownian motion and behave as rigid magnetic dipoles, while the magnetization of the carrier liquid had been taken into account from its general equilibrium expression. We used a special case of a general three-dimensional theoretical model that governs the micropolar hydrodynamic flow in such liquids, which was based on the reduction of the partial differential equations to a simpler shape that is similar to low-Reynolds number flow and are called magnetic Stokes equations. The interaction of the applied magnetic field with the ferromagnetic particles has been inherited within those equations through the additional effective viscosity and the magnetic pressure of the fluid in terms of both its hydrodynamic and magnetic properties.

We used an improved new complete and unique differential representation of magnetic Stokes flow, drawn from the potential representation theory, which is valid for nonaxisymmetric micropolar flows and provides in an analytical fashion the velocity and total pressure fields in terms of easy-to-find potentials. Therein, we applied the proper boundary conditions according to the physical requirements on the boundaries, and we managed in a cumbersome and tedious way to obtain the three-dimensional magnetic Stokes flow fields in a closed analytical form of expansions of infinite series, in terms of the applied 3D constant magnetic field, of the interior circular cylindrical eigensolutions, and of the certain hydrodynamic or magnetic parameters.

In order to study the characteristics of the 3D flow and compute the velocity and total pressure fields associated with creeping magnetic flow, we proceeded to a necessary numerical implementation of our results in a circular duct. The development of the flow, as the magnetic fluid moves downstream of the duct under the effect of the imposed magnetic field, has been presented graphically for various imposed velocities at the inlet, while the variation of the additional viscosity due to the magnetic field versus the magnetic field magnitude and the concentration of particles inside the ferrofluid has been depicted through specific plots.

\section{Appendix}

\section{Mathematical Material}

In the interest of making this paper complete and independent it is necessary to provide some useful information concerning important mathematical tools, which are adequate for both the analytical and the numerical part of the present paper. 


\section{Differential Identities}

Consequently, we begin by providing important identities [29], which were used extensively during our analytical calculations. Let $u, v$ and $\mathbf{f}, \mathbf{g}$ denote two scalar and two vector fields, respectively. Then, the basic vector differential identities involve the action of the gradient and the Laplace operators on certain expressions, that is,

$$
\begin{gathered}
\nabla(u v)=u \nabla v+v \nabla u, \\
\nabla \otimes(u \mathbf{f})=u(\nabla \otimes \mathbf{f})+\nabla u \otimes \mathbf{f}, \\
\nabla \cdot(u \mathbf{f})=u \nabla \cdot \mathbf{f}+\nabla u \cdot \mathbf{f}, \\
\nabla \times(u \mathbf{f})=u \nabla \times \mathbf{f}+\nabla u \times \mathbf{f}, \\
\nabla(\mathbf{f} \cdot \mathbf{g})=(\nabla \otimes \mathbf{f}) \cdot \mathbf{g}+(\nabla \otimes \mathbf{g}) \cdot \mathbf{f}, \\
\nabla \cdot(\mathbf{f} \otimes \mathbf{g})=(\nabla \cdot \mathbf{f}) \mathbf{g}+\mathbf{f} \cdot(\nabla \otimes \mathbf{g}), \\
\nabla \times \nabla \times \mathbf{f}=\nabla(\nabla \cdot \mathbf{f})-\Delta \mathbf{f}, \\
\nabla \cdot(\nabla \otimes \mathbf{f})=\Delta \mathbf{f}, \\
\nabla \cdot(\nabla \otimes \mathbf{f})^{\mathrm{T}}=\nabla(\nabla \cdot \mathbf{f}), \\
\Delta(u \mathbf{f})=\mathbf{f} \Delta u+u \Delta \mathbf{f}+2 \nabla u \cdot(\nabla \otimes \mathbf{f}), \\
\Delta(\mathbf{f} \cdot \mathbf{g})=\mathbf{f} \cdot \Delta \mathbf{g}+\mathbf{g} \cdot \Delta \mathbf{f}+2(\nabla \otimes \mathbf{f})^{\mathrm{T}}:(\nabla \otimes \mathbf{g}),
\end{gathered}
$$

where the symbols " $\otimes$ ", ":", and "T" denote juxtaposition, double inner product, and transposition, respectively. The proofs of identities (A.1)-(A.11) are based on classical analysis by expanding the vectors $\mathbf{f}$ and $\mathbf{g}$ in Cartesian coordinates.

\section{Circular Cylindrical Coordinate System}

In terms of the three variables $r \in[0,+\infty), \varphi \in[0,2 \pi)$, and $z \in(-\infty,+\infty)$, we define the implemented to our work circular cylindrical coordinate system [27] via the Cartesian basis as follows:

$$
\mathbf{r}=\sum_{i=1}^{3} x_{i} \widehat{\mathbf{x}}_{i}=z \widehat{\mathbf{x}}_{1}+r \cos \varphi \widehat{\mathbf{x}}_{2}+r \sin \varphi \widehat{\mathbf{x}}_{3}
$$

where $\mathbf{r}$ is the position vector, while the coordinate vectors of this system $\widehat{\mathbf{r}}, \widehat{\boldsymbol{\varphi}}, \widehat{\mathbf{z}}$ assume the forms

$$
\widehat{\mathbf{r}}=-\frac{\partial \widehat{\boldsymbol{\varphi}}}{\partial \varphi}=\cos \varphi \widehat{\mathbf{x}}_{2}+\sin \varphi \widehat{\mathbf{x}}_{3}, \quad \widehat{\boldsymbol{\varphi}}=\frac{\partial \widehat{\mathbf{r}}}{\partial \varphi}=-\sin \varphi \widehat{\mathbf{x}}_{2}+\cos \varphi \widehat{\mathbf{x}}_{3}, \quad \widehat{\mathbf{z}}=\widehat{\mathbf{x}}_{1}
$$


or their inverse

$$
\widehat{\mathbf{x}}_{1}=\widehat{\mathbf{z}}, \quad \widehat{\mathbf{x}}_{2}=\cos \varphi \widehat{\mathbf{r}}-\sin \varphi \widehat{\boldsymbol{\varphi}}, \quad \widehat{\mathbf{x}}_{3}=\sin \varphi \widehat{\mathbf{r}}+\cos \varphi \widehat{\boldsymbol{\varphi}} .
$$

The gradient $\nabla$ and the Laplacian $\Delta$ are provided by the expressions

$$
\nabla=\sum_{i=1}^{3} \widehat{\mathbf{x}}_{i} \frac{\partial}{\partial x_{i}}=\widehat{\mathbf{r}} \frac{\partial}{\partial r}+\frac{\widehat{\boldsymbol{\varphi}}}{r} \frac{\partial}{\partial \varphi}+\widehat{\mathbf{z}} \frac{\partial}{\partial z}, \quad \Delta=\sum_{i=1}^{3} \frac{\partial^{2}}{\partial x_{i}^{2}}=\frac{1}{r} \frac{\partial}{\partial r}\left(r \frac{\partial}{\partial r}\right)+\frac{1}{r^{2}} \frac{\partial^{2}}{\partial \varphi^{2}}+\frac{\partial^{2}}{\partial z^{2}},
$$

and then the forthcoming relationships are easily verified using the vector relations (A.12), (A.13), and (A.15), that is,

$$
\nabla \otimes \widehat{\mathbf{r}}=\frac{1}{r} \widehat{\boldsymbol{\varphi}} \otimes \widehat{\boldsymbol{\varphi}}, \quad \nabla \otimes \widehat{\boldsymbol{\varphi}}=-\frac{1}{r} \widehat{\boldsymbol{\varphi}} \otimes \widehat{\mathbf{r}}, \quad \nabla \otimes \widehat{\mathbf{z}}=\tilde{\mathbf{0}},
$$

as well as

$$
\nabla \otimes \mathbf{r}=\widetilde{\mathbf{I}}=\sum_{i=1}^{3} \widehat{\mathbf{x}}_{i} \otimes \widehat{\mathbf{x}}_{i}=\widehat{\mathbf{r}} \otimes \widehat{\mathbf{r}}+\widehat{\boldsymbol{\varphi}} \otimes \widehat{\boldsymbol{\varphi}}+\widehat{\mathbf{z}} \otimes \widehat{\mathbf{z}}
$$

whereas $\widetilde{I}$ stands for the unit dyadic and $\tilde{\mathbf{0}}$ refers to the zero dyadic [28]. Our boundary value problem is adjusted to the type of circular cylindrical geometry introduced here, where the $x_{1}$-axis is the axis of symmetry of an infinite circular cylinder and the other two axes are located properly so as to obtain a $(r, \varphi, z)$ right-handed system.

\section{Bessel and Neumann Special Functions}

In the sequel, we define the special functions used in this project. The Bessel functions $J_{n}(x)$ and the Neumann functions $N_{n}(x)$ [29] of order $n \geq 0$ are linear independent solutions of the Bessel differential equation

$$
x^{2} y^{\prime \prime}(x)+x y^{\prime}(x)+\left(x^{2}-n^{2}\right) y(x)=0,
$$

for $n=0,1,2, \ldots$, which is valid for every $x \in \mathbb{R}$. These functions are defined as follows:

$$
\begin{gathered}
J_{n}(x)=\sum_{\kappa=0}^{\infty} \frac{(-1)^{\kappa}}{\kappa !(n+\kappa) !}\left(\frac{x}{2}\right)^{n+2 \kappa} \quad \text { for } x \in \mathbb{R}, \\
N_{n}(x)=\frac{1}{\pi}\left[\frac{d J_{v}(x)}{d v}-(-1)^{n} \frac{d J_{-v}(x)}{d v}\right]_{v=n} \quad \text { for } x \in \mathbb{R},
\end{gathered}
$$

for $n \geq 0$ and $v \in \mathbb{R}$, where the Bessel functions $J_{v}(x)$ for real $v$ are furnished by the formula

$$
J_{v}(x)=\sum_{\kappa=0}^{\infty} \frac{(-1)^{\kappa}}{\kappa ! \Gamma(\kappa+v+1)}\left(\frac{x}{2}\right)^{v+2 \kappa} \quad \text { with } \Gamma(\kappa+v+1)=\int_{0}^{+\infty} e^{-t} t^{\kappa+v} d t \text { for } x \in \mathbb{R}
$$


via the well-known Gamma function $\Gamma(\kappa+v+1)$ [29] for every $\kappa \geq 0$ and $v \in \mathbb{R}$. In our case, where $v=n=0,1,2, \ldots$, it is easily confirmed that $J_{-n}(x)=(-1)^{n} J_{n}(x)$ and $N_{-n}(x)=$ $(-1)^{n} N_{n}(x)$ for $x \in \mathbb{R}$. The limiting cases as $x \rightarrow 0$, where $J_{n}(x)$ converge, or as $x \rightarrow+\infty$, where $N_{n}(x)$ are regular, are discussed in many standard references such as [29], and it is not worth to mention them here. The Bessel and the Neumann functions satisfy the important recurrence relations

$$
\begin{aligned}
\frac{d Z_{n}(x)}{d x}=\frac{1}{2}\left[Z_{n-1}(x)-Z_{n+1}(x)\right] & =\frac{n}{x} Z_{n}(x)-Z_{n+1}(x)=Z_{n-1}(x)-\frac{n}{x} Z_{n}(x) \quad \text { for } x \in \mathbb{R}, \\
\frac{2 n}{x} Z_{n}(x) & =Z_{n-1}(x)+Z_{n+1}(x) \quad \text { for } x \in \mathbb{R}
\end{aligned}
$$

defined as $Z_{n}(x) \equiv J_{n}(x)$ or $Z_{n}(x) \equiv N_{n}(x)$ for $n \geq 0$ and $x \in \mathbb{R}$. The Bessel differential equation (A.18) and its solutions (A.19)-(A.21) appear in several physical boundary value problems such as ours, where under the same notation the variable $x$ is written as $x=\mu r \in$ $\mathbb{R}$, with $\mu \in \mathbb{R}$ being a constant parameter, which comes from the method of separation of variables of a partial differential equation in the circular cylindrical coordinate system. This parameter is specified by the boundary conditions of the particular physical problem that has to be satisfied in each case. Upon that procedure, if the parameter $\mu$ becomes an eigenvalue $\mu_{n}^{m} \in \mathbb{R}$ for $n \geq 0$ and $m \geq 1$, then $x$ is substituted by $x=\mu r \equiv \mu_{n}^{m} r \in \mathbb{R}$, whereas $\mu \equiv \mu_{n}^{m}$ is the $m$-parameter $(m \geq 1)$ of order $n \geq 0$. Thus, both the Bessel and the Neumann functions satisfy the orthogonality relation

$$
\begin{aligned}
\int_{a}^{b} r Z_{n}\left(\mu_{n}^{m^{\prime}} r\right) Z_{n}\left(\mu_{n}^{m} r\right) d r=\delta_{m m^{\prime}} & \frac{b^{2}}{2}\left[\left(Z_{n}\left(\mu_{n}^{m} b\right)\right)^{2}-Z_{n-1}\left(\mu_{n}^{m} b\right) Z_{n+1}\left(\mu_{n}^{m} b\right)\right] \\
& \left.-\frac{a^{2}}{2}\left[\left(Z_{n}\left(\mu_{n}^{m} a\right)\right)^{2}-Z_{n-1}\left(\mu_{n}^{m} a\right) Z_{n+1}\left(\mu_{n}^{m} a\right)\right]\right\},
\end{aligned}
$$

for $n \geq 0$ and $m, m^{\prime} \geq 1$, with $\delta_{m m^{\prime}}$ being the Kronecker delta and $a, b \in \mathbb{R}$ being constants.

\section{Trigonometric and Hyperbolic Functions}

As far as the trigonometric functions $\sin n \varphi$ and $\cos n \varphi$ are concerned for $n \geq 0$, which are used in this paper, the following expressions hold true:

$$
\begin{aligned}
\sin \varphi \sin n \varphi & =\frac{1}{2}[\cos (n-1) \varphi-\cos (n+1) \varphi], \\
\cos \varphi \cos n \varphi & =\frac{1}{2}[\cos (n-1) \varphi+\cos (n+1) \varphi], \\
\cos \varphi \sin n \varphi & =\frac{1}{2}[\sin (n+1) \varphi+\sin (n-1) \varphi], \\
\sin \varphi \cos n \varphi & =\frac{1}{2}[\sin (n+1) \varphi-\sin (n-1) \varphi],
\end{aligned}
$$


where $\varphi \in[0,2 \pi)$ stands for the azimuthal angle, taken for the first period of the trigonometric circle, while the orthogonality here is obvious for the functions $\sin n \varphi$ and $\cos n \varphi$ for $n \geq 0$. On the other hand, the hyperbolic functions introduced in our paper are well known and can be taken also by their exponential form, that is,

$$
\sinh (\mu z)=\frac{e^{\mu z}-e^{-\mu z}}{2}, \quad \cosh (\mu z)=\frac{e^{\mu z}+e^{-\mu z}}{2} \text { with } \tanh (\mu z)=\frac{1}{\operatorname{coth}(\mu z)}=\frac{\sinh (\mu z)}{\cosh (\mu z)}
$$

where $z \in(-\infty,+\infty)$, whilst the parameter $\mu$, as we mentioned previously, comes from the boundary conditions of a specific boundary value problem.

\section{Harmonic Functions in Circular Cylindrical Geometry}

Then, in view of our physical problem, every harmonic function $w(\Delta w(r, \varphi, z)=0)$ in the circular cylindrical coordinate system is written as

$$
w(r, \varphi, z)=\sum_{n=0}^{\infty} \int \sum_{\mu}\left[a_{n}^{\mu} J_{n}(\mu r)+b_{n}^{\mu} N_{n}(\mu r)\right]\left(c_{n}^{\mu} \cos n \varphi+d_{n}^{\mu} \sin n \varphi\right)\left(e_{n}^{\mu} \cosh (\mu z)+f_{n}^{\mu} \sinh (\mu z)\right)
$$

where $a_{n}^{\mu}, b_{n}^{\mu}, c_{n}^{\mu}, d_{n}^{\mu}, e_{n}^{\mu}$, and $f_{n}^{\mu}$ for $n \geq 0$ are constants, whilst the symbol " $\int \Sigma_{\mu} \ldots$ " refers to the constant parameter $\mu$ and denotes integration if $\mu$ takes continuous values or summation in the case where $\mu$ is a parameter with discrete values. At this point we must make the following basic remark. Here, as we pointed earlier, the parameter $\mu$ comes from the method of separation of variables for the Laplace equation in circular cylindrical coordinates, where only the particular physical problem and the boundary conditions can dictate its value. For example, as in our case, when one has to face an interior problem inside a circular cylinder of radius $\alpha \in \mathbb{R}$, where the boundary conditions require nihilism of the Bessel functions on the surface of the cylinder, then the regularity of the solution demands $Z_{n}(\mu r) \equiv J_{n}(\mu r)$ and the boundary conditions provide us with the discrete values of the parameter as $\mu \equiv \mu_{n}^{m}=\rho_{n}^{m} / \alpha$, where $\rho_{n}^{m} \in \mathbb{R}$ is the $m$-root $(m \geq 1)$ of order $n \geq 0$ of the Bessel functions and the eigenvalue now $\mu_{n}^{m}$ is the $m$-parameter $(m \geq 1)$ of order $n \geq 0$. Hence, for this particular problem of determining parameter $\mu$, expansion (A.30) becomes

$$
\begin{aligned}
w(r, \varphi, z)=\sum_{n=0}^{\infty} \sum_{m=1}^{\infty} & {\left[a_{n}^{m} J_{n}\left(\mu_{n}^{m} r\right)+b_{n}^{m} N_{n}\left(\mu_{n}^{m} r\right)\right]\left(c_{n}^{m} \cos n \varphi+d_{n}^{m} \sin n \varphi\right) } \\
\times & \times\left(e_{n}^{m} \cosh \left(\mu_{n}^{m} z\right)+f_{n}^{m} \sinh \left(\mu_{n}^{m} z\right)\right)
\end{aligned}
$$

where $a_{n}^{m}, b_{n}^{m}, c_{n}^{m}, d_{n}^{m}, e_{n}^{m}$, and $f_{n}^{m}$ for $n \geq 0, m \geq 1$ are the new constants, while we have $a=0, b=\alpha$ and relation (A.24) takes the form

$$
\int_{0}^{\alpha} r J_{n}\left(\rho_{n}^{m^{\prime}} \frac{r}{\alpha}\right) J_{n}\left(\rho_{n}^{m} \frac{r}{\alpha}\right) d r=\delta_{m m^{\prime}} \frac{\alpha^{2}}{2}\left(J_{n+1}\left(\rho_{n}^{m}\right)\right)^{2}, \quad n \geq 0, m, m^{\prime} \geq 1,
$$


which is the orthogonality expression frequently utilized within our calculations. In addition, this situation of an internal problem requires the use of regular solutions on the axis of symmetry of the circular cylinder $(r=0)$, which means that only the Bessel functions $J_{n}(\mu r) \equiv J_{n}\left(\mu_{n}^{m} r\right)$ survive inside the expansion (A.30) or (A.31), and, thus, this is reflected to the vanishing of the corresponding constants, that is, $b_{n}^{\mu}=0$ for $n \geq 0$ or $b_{n}^{m}=0$ for $n \geq 0$ and $m \geq 1$, respectively.

\section{References}

[1] B. Berkovski and V. Bashtovoy, Magnetic Fluids and Applications Handbook, Begell House, New York, NY, USA, 1996.

[2] R. W. Fox and A. T. McDonald, Introduction to Fluid Mechanics, John Wiley \& Sons, New York, NY, USA, 1985.

[3] V. G. Bashotovoy, B. M. Berkovski, and A. N. Vislovich, Introduction to Thermomechanics of Magnetic Fluids, Hemisphere, New York, NY, USA, 1988.

[4] R. E. Rosensweig, Ferrohydrodynamics, Dover, New York, NY, USA, 1997.

[5] L. D. Landau, E. M. Lifshitz, and L. P. Pitaevskii, Electrodynamics of Continuous Media, Pergamon Press, New York, NY, USA, 1984.

[6] P. M. Hatzikonstantinou and P. Vafeas, "A general theoretical model for the magnetohydrodynamic flow of micropolar magnetic fluids. Application to Stokes flow," Mathematical Methods in the Applied Sciences, vol. 33, no. 2, pp. 233-248, 2010.

[7] J. Happel and H. Brenner, Low Reynolds Number Hydrodynamics with Special Applications to Particulate Media, Prentice-Hall, Englewood Cliffs, NJ, USA, 1986.

[8] H. Neuber, "Ein neuer ansatz zur lösung räumblicher probleme der elastizitätstheorie," Journal of Applied Mathematics and Mechanics, vol. 14, pp. 203-212, 1934.

[9] P. M. Naghdi and C. S. Hsu, "On a representation of displacements in linear elasticity in terms of three stress functions," Journal of Mathematical Mechanics, vol. 10, pp. 233-245, 1961.

[10] X. S. Xu and M. Z. Wang, "General complete solutions of the equations of spatial and axisymmetric Stokes flow," The Quarterly Journal of Mechanics and Applied Mathematics, vol. 44, no. 4, pp. 537-548, 1991.

[11] P. D. S. Verma, “On a class of exact solutions to magnetic fluid equations," Journal of Magnetism and Magnetic Materials, vol. 39, no. 1-2, pp. 137-141, 1983.

[12] V. U. K. Sastry and V. R. Mohan Rao, "Micropolar fluid flow due to an oscillating plane subject to rotation," Acta Mechanica, vol. 33, no. 1-2, pp. 45-53, 1979.

[13] C. Calmelet-Eluhu and D. R. Majumdar, "Flow of a micropolar fluid through a circular cylinder subject to longitudinal and torsional oscillations," Mathematical and Computer Modelling, vol. 27, no. 8, pp. $69-78,1998$.

[14] H. C. Weng, C.-K. Chen, and M.-H. Chang, "Stability of micropolar fluid flow between concentric rotating cylinders," Journal of Fluid Mechanics, vol. 631, pp. 343-362, 2009.

[15] M. S. Faltas and E. I. Saad, "Stokes flow with slip caused by the axisymmetric motion of a sphere bisected by a free surface bounding a semi-infinite micropolar fluid," International Journal of Engineering Science, vol. 43, no. 11-12, pp. 953-976, 2005.

[16] H. H. Sherif, M. S. Faltas, and E. I. Saad, "Slip at the surface of a sphere translating perpendicular to a plane wall in micropolar fluid," Zeitschrift für Angewandte Mathematik und Physik, vol. 59, no. 2, pp. 293-312, 2008.

[17] A. Moosaie and G. Atefi, "Slow motion of a rotating circular cylinder through a micropolar fluid," Archives of Mechanics, vol. 60, no. 3, pp. 199-220, 2008.

[18] P. K. Papadopoulos and E. E. Tzirtzilakis, "Biomagnetic flow in a curved square duct under the influence of an applied magnetic field," Physics of Fluids, vol. 16, no. 8, pp. 2952-2962, 2004.

[19] S. A. Khashan and Y. Haik, "Numerical simulation of biomagnetic fluid downstream an eccentric stenotic orifice," Physics of Fluids, vol. 18, no. 11, Article ID 113601, 10 pages, 2006.

[20] C. C. Sellers and J. S. Walker, "Liquid-metal flow in an electrically insulated rectangular duct with a non-uniform magnetic field," International Journal of Engineering Science, vol. 37, no. 5, pp. 541-552, 1999.

[21] L. M. Witkowski and J. S. Walker, "Numerical solutions for the liquid-metal flow in a rotating cylinder with a weak transverse magnetic field," Fluid Dynamics Research, vol. 30, no. 3, pp. 127-137, 2002. 
[22] P. K. Papadopoulos, P. Vafeas, and P. M. Hatzikonstantinou, "Numerical study of a new model for the magnetohydrodynamic flow of micropolar magnetic fluids in straight square ducts," in Proceedings of the 6th International Conference on Engineering Computational Technology, pp. 1-19, Civil-Comp Press, 2008.

[23] P. Vafeas, P. K. Papadopoulos, and P. M. Hatzikonstantinou, "Micropolar flow under the effect of a magnetic dipole," in Proceedings of the 6th International Conference of Computational Methods in Sciences and Engineering, vol. 1148 of American Institute of Physics Proceedings, pp. 566-570, 2008.

[24] G. Dassios, M. Hadjinicolaou, and A. C. Payatakes, "Generalized eigenfunctions and complete semiseparable solutions for Stokes flow in spheroidal coordinates," Quarterly of Applied Mathematics, vol. 52, no. 1, pp. 157-191, 1994.

[25] P. Vafeas and G. Dassios, "Stokes flow in ellipsoidal geometry," Journal of Mathematical Physics, vol. 47, no. 9, Article ID 093102, 38 pages, 2006.

[26] L. Martin Witkowski, J. S. Walker, and P. Marty, "Nonaxisymmetric flow in a finite-length cylinder with a rotating magnetic field," Physics of Fluids, vol. 11, no. 7, pp. 1821-1826, 1999.

[27] P. Moon and E. Spencer, Field Theory Handbook, Springer, Berlin, Germany, 1971.

[28] P. M. Morse and H. Feshbach, Methods of Theoretical Physics, Vol I, II, McGraw-Hill, New York, NY, USA, 1953.

[29] E. W. Hobson, The Theory of Spherical and Ellipsoidal Harmonics, Chelsea, New York, NY, USA, 1965.

[30] P. M. Gresho, "On the theory of semi-implicit projection methods for viscous incompressible flow and its implementation via a finite element method that also introduces a nearly consistent mass matrix. I. Theory," International Journal for Numerical Methods in Fluids, vol. 11, no. 5, pp. 587-620, 1990.

[31] R. K. Ganesh, "Evaluation of the drag force by integrating the energy dissipation rate in Stokes flow for 2D domains using the FEM," International Journal for Numerical Methods in Fluids, vol. 13, no. 5, pp. 557-578, 1991.

[32] C. H. Bruneau and P. Fabrie, "Effective downstream boundary conditions for incompressible NavierStokes equations," International Journal for Numerical Methods in Fluids, vol. 19, no. 8, pp. 693-705, 1994.

[33] G. B. Arfken and H. J. Weber, Mathematical Methods for Physicists, Elsevier Academic, New York, NY, USA, 5th edition, 2005.

[34] R. A. Eubanks and E. Sternberg, "On the completeness of the Boussinesq-Papkovich stress functions," Journal of Rational Mechanics and Analysis, vol. 5, pp. 735-746, 1956.

[35] W. M. Rohsenow, J. P. Hartnett, and Y. I. Cho, Handbook of Heat Transfer, McGraw-Hill, New York, NY, USA, 1998. 


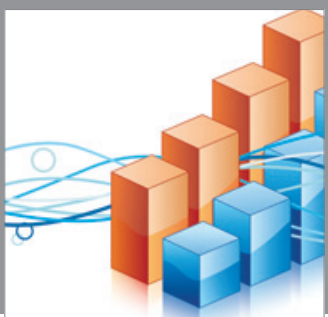

Advances in

Operations Research

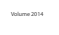

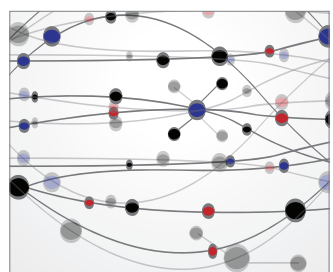

\section{The Scientific} World Journal
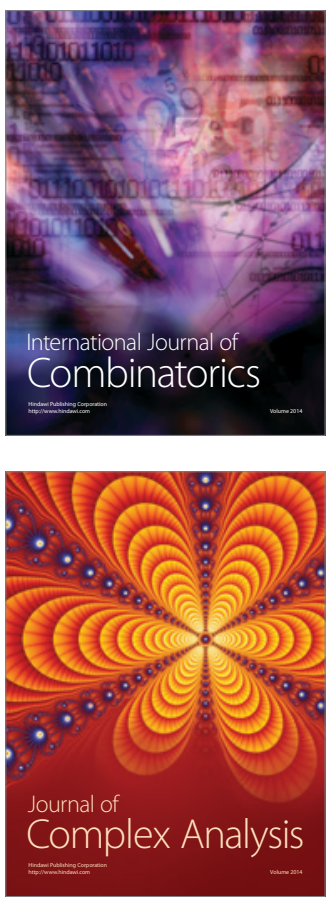

International Journal of

Mathematics and

Mathematical

Sciences
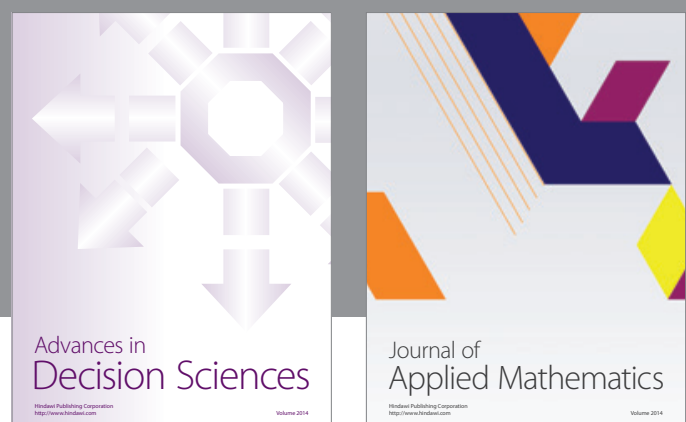

Journal of

Applied Mathematics
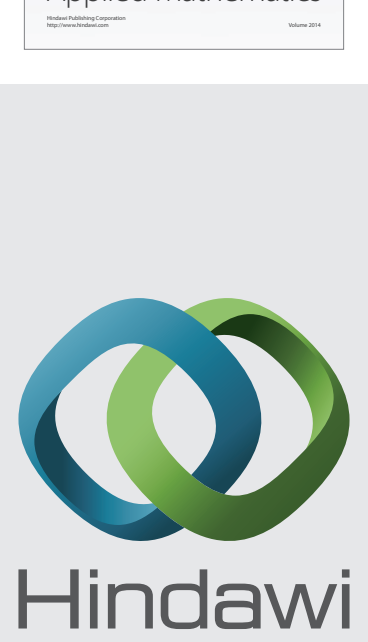

Submit your manuscripts at http://www.hindawi.com
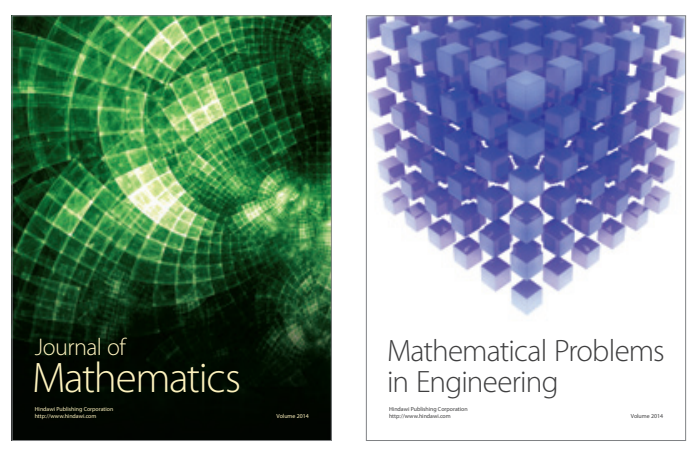

Mathematical Problems in Engineering
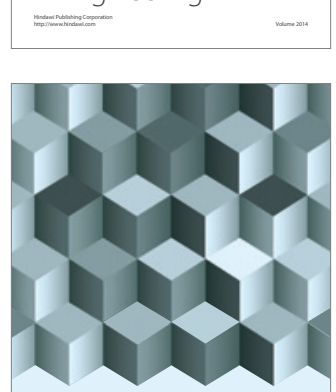

Journal of

Function Spaces
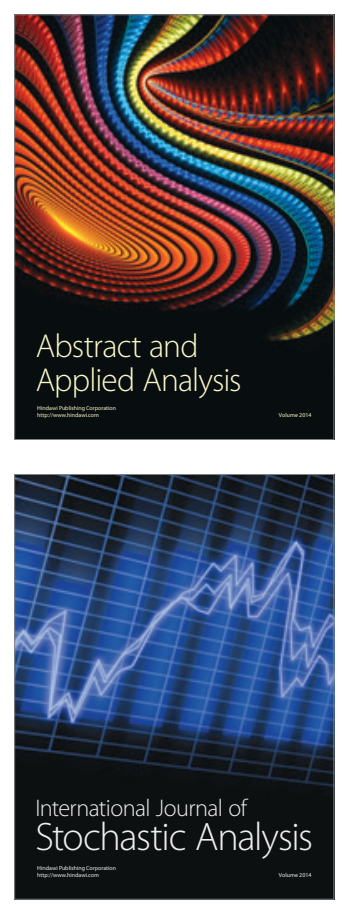

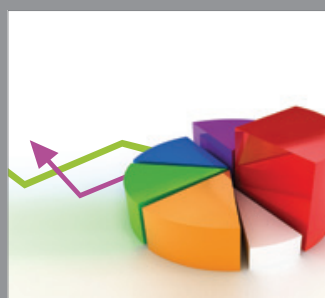

ournal of

Probability and Statistics

Promensencen
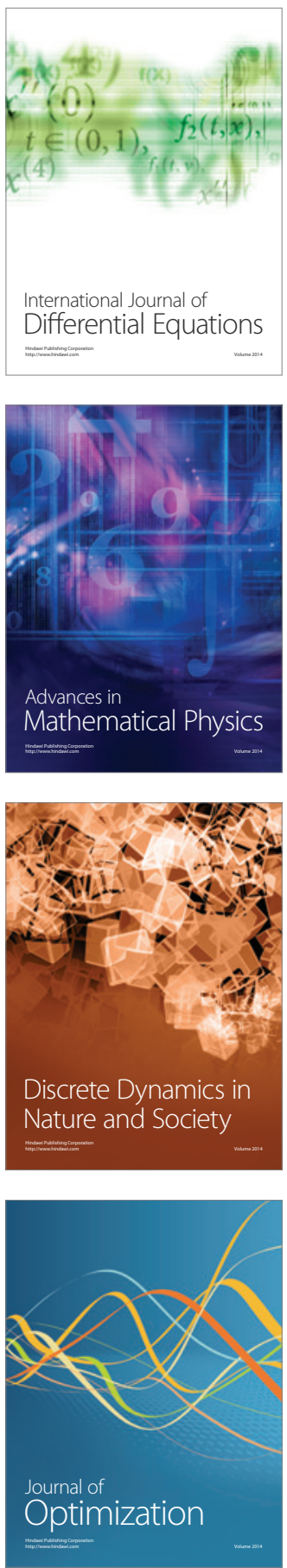